\title{
VHE $\gamma$-RAY OBSERVATION OF THE CRAB NEBULA AND ITS PULSAR WITH THE MAGIC TELESCOPE
}

J. Albert ${ }^{1}$ E. Aliu,${ }^{2}$ H. Anderhub,${ }^{3}$ P. Antoranz ${ }^{4}$ A. Armada,${ }^{2}$ C. Baixeras,${ }^{5}$ J. A. Barrio, ${ }^{4}$ H. Bartko, ${ }^{6}$ D. Bastieri, ${ }^{7}$ J. K. Becker, ${ }^{8}$ W. Bednarek, ${ }^{9}$ K. Berger, ${ }^{1}$ C. Bigongiari, ${ }^{7}$ A. Biland, ${ }^{3}$ R. K. Bock, ${ }^{6,7}$ P. Bordas, ${ }^{10}$ V. Bosch-Ramon, ${ }^{10}$ T. Bretz, ${ }^{1}$ I. Britvitch, ${ }^{3}$ M. Camara, ${ }^{4}$ E. Carmona, ${ }^{6}$ A. Chilingarian, ${ }^{11}$ J. A. Coarasa, ${ }^{6}$ S. Commichau, ${ }^{3}$ J. L. Contreras, ${ }^{4}$ J. Cortina, ${ }^{2}$ M. T. Costado, ${ }^{12}$ V. Curtef, ${ }^{8}$ V. Danielyan, ${ }^{11}$ F. Dazzi, ${ }^{7}$ A. De Angelis, ${ }^{13}$ C. Delgado, ${ }^{12}$ R. de los Reyess ${ }_{4}$ B. De Lotto, ${ }^{13}$ E. Domingo-Santamaría, ${ }^{2}$ D. Dorner, ${ }^{1}$ M. Doro, ${ }^{7}$ M. Errando, ${ }^{2}$ M. Fagiolini, ${ }^{14}$ D. Ferenc, ${ }^{15}$ E. Fernández, ${ }^{2}$ R. Firpo, ${ }^{2}$ J. Flix, ${ }^{2}$ M. V. Fonseca, ${ }^{4}$ L. Font, ${ }^{5}$ M. Fuchs, ${ }^{6}$ N. Galante, ${ }^{6}$ R. García-López, ${ }^{12}$ M. Garczarczyk, ${ }^{6}$ M. Gaug, ${ }^{7}$ M. Giller, ${ }^{9}$ F. Goebel, ${ }^{6}$ D. Hakobyan, ${ }^{11}$ M. Hayashida, ${ }^{6}$ T. Hengstebeck, ${ }^{16}$ A. Herrero, ${ }^{12}$ D. Höhne, ${ }^{1}$ J. Hose, ${ }^{6}$ C. C. Hsu, ${ }^{6}$ P. Jacon,${ }^{9}$ T. Jogler,${ }^{6}$ R. Kosyra, ${ }^{6}$ D. Kranich, ${ }^{3}$ R. Kritzer, ${ }^{1}$ A. Laille, ${ }^{15}$ E. Lindfors, ${ }^{17}$ S. Lombardi, ${ }^{7}$ F. Longo, ${ }^{13}$ J. López, ${ }^{2}$ M. López, ${ }^{4}$ E. Lorenz, ${ }^{3,6}$ P. Majumdar, ${ }^{6}$ G. Maneva, ${ }^{18}$ K. Mannheim, ${ }^{1}$ O. Mansutti ${ }^{13}$ M. Mariotti, ${ }^{7}$ M. Martínez, ${ }^{2}$ D. Mazin, ${ }^{6}$ C. Merck, ${ }^{6}$ M. Meucci, ${ }^{14}$ M. Meyer, ${ }^{1}$ J. M. Miranda, ${ }^{4}$ R. Mirzoyan, ${ }^{6}$ S. Mizobuchi, ${ }^{6}$ A. Moralejo, ${ }^{2}$ D. Nieto, ${ }^{4}$ K. Nilsson, ${ }^{17}$ J. Ninkovic, ${ }^{6}$ E. OÑa-Wilhelmi, ${ }^{2}$ N. Otte, ${ }^{6,16}{ }^{14}$ Oya, ${ }^{4}$ D. Paneque, ${ }^{6}$ M. Panniello, ${ }^{12}$ R. Paoletti, ${ }^{14}$ J. M. Paredes, ${ }^{10}$ M. Pasanen, ${ }^{17}$ D. Pascoli, ${ }^{7}$ F. Pauss,${ }^{3}$ R. Pegna, ${ }^{14}$ M. Persic,${ }^{13,19}$ L. Peruzzo, ${ }^{7}$ A. Piccioli, ${ }^{14}$ M. Poller, ${ }^{1}$ E. Prandini, ${ }^{7}$ N. Puchades, ${ }^{2}$ A. Raymers, ${ }^{11}$ W. Rhode, ${ }^{8}$ M. Ribó,,${ }^{10}$ J. Rico, ${ }^{2}$ M. Rissi, ${ }^{3}$ A. Robert, ${ }^{5}$ S. Rügamer, ${ }^{1}$ A. SAggion, ${ }^{7}$ A. SÁnchez, ${ }^{5}$ P. Sartori, ${ }^{7}$ V. Scalzotto, ${ }^{7}$ V. Scapin, ${ }^{13}$ R. Schmitt, ${ }^{1}$ T. Schweizer, ${ }^{6}$ M. Shayduk, ${ }^{6,16}$ K. Shinozaki, ${ }^{6}$ S. N. Shore, ${ }^{20}$ N. Sidro, ${ }^{2}$ A. Sillanpää, ${ }^{17}$ D. Sobczynska, ${ }_{18}^{9}$ A. Stamerra, ${ }^{14}$ L. S. Stark, ${ }^{3}$ L. Takalo, ${ }_{6} 17$ P. Temnikov, ${ }^{18}$ D. Tescaro, ${ }^{2}$ M. Teshima, ${ }^{6}$ N. Tonello, ${ }^{6}$ D. F. Torres, ${ }^{2,21}$ N. Turini, ${ }^{14}$ H. Vankov, ${ }^{18}$ V. Vitale, ${ }^{13}$ R. M. Wagner, ${ }^{6}$ T. Wibig, ${ }^{9}$ W. Wittek, ${ }^{6}$ F. Zandanel, ${ }^{7}$ R. Zanin, ${ }^{2}$ AND J. ZaPATERO ${ }^{5}$ Received 2007 May 18; accepted 2007 October 31

\begin{abstract}
We report about very high energy (VHE) $\gamma$-ray observations of the Crab Nebula with the MAGIC telescope. The $\gamma$-ray flux from the nebula was measured between $60 \mathrm{GeV}$ and $9 \mathrm{TeV}$. The energy spectrum can be described by a curved power law $d F / d E=f_{0}(E / 300 \mathrm{GeV})^{\left[a+b \log _{10}(E / 300 \mathrm{GeV})\right]}$ with a flux normalization $f_{0}$ of $\left(6.0 \pm 0.2_{\text {stat }}\right) \times 10^{-10} \mathrm{~cm}^{-2} \mathrm{~s}^{-1}$ $\mathrm{TeV}^{-1}, a=-2.31 \pm 0.06_{\text {stat }}$, and $b=-0.26 \pm 0.07_{\text {stat }}$. The peak in the spectral energy distribution is estimated at $77 \pm 35 \mathrm{GeV}$. Within the observation time and the experimental resolution of the telescope, the $\gamma$-ray emission is steady and pointlike. The emission's center of gravity coincides with the position of the pulsar. Pulsed $\gamma$-ray emission from the pulsar could not be detected. We constrain the cutoff energy of the pulsed spectrum to be less than $27 \mathrm{GeV}$, assuming that the differential energy spectrum has an exponential cutoff. For a superexponential shape, the cutoff energy can be as high as $60 \mathrm{GeV}$.
\end{abstract}

Subject headings: acceleration of particles — gamma rays: observations — pulsars: individual (PSR B0531+21) radiation mechanisms: nonthermal

\section{INTRODUCTION}

The Crab Nebula is the remnant of a supernova explosion that occurred in AD 1054 (e.g., Collins et al. 1999 and references therein) at a distance of $\sim 2 \mathrm{kpc}$. It is one of the best-studied nonthermal celestial objects in almost all wavelength bands of

1 Universität Würzburg, D-97074 Würzburg, Germany.

2 Institut de Física d'Altes Energies, E-08193 Bellaterra (Barcelona), Spain.

3 ETH Zurich, CH-8093 Zurich, Switzerland.

4 Universidad Complutense, E-28040 Madrid, Spain.

5 Universitat Autònoma de Barcelona, E-08193 Bellaterra, Spain.

6 Max-Planck-Institut für Physik, D-80805 München, Germany.

7 Università di Padova and INFN, I-35131 Padova, Italy.

8 Universität Dortmund, D-44227 Dortmund, Germany.

9 University of Łódź, PL-90236 Lodz, Poland.

${ }^{10}$ Universitat de Barcelona, E-08028 Barcelona, Spain.

11 Yerevan Physics Institute, AM-375036 Yerevan, Armenia.

12 Instituto de Astrofisica de Canarias, E-38200, La Laguna, Tenerife, Spain. the electromagnetic spectrum from $10^{-5} \mathrm{eV}$ (radio) to nearly $10^{14} \mathrm{eV}(\gamma$-rays). There is little doubt that the engine of the nebula is the pulsar PSR B0531+21 (hereafter Crab pulsar) in its center.

In very high energy ( VHE) $\gamma$-ray astronomy the Crab Nebula was first detected with large significance at TeV energies by the pioneering Whipple telescope (Weekes et al. 1989). The nebula

\footnotetext{
13 Università di Udine and INFN Trieste, I-33100 Udine, Italy.

14 Università di Siena and INFN Pisa, I-53100 Siena, Italy.

15 University of California, Davis, CA 95616-8677.

16 Humboldt-Universität zu Berlin, D-12489 Berlin, Germany.

17 Tuorla Observatory, Turku University, FI-21500 Piikkiö, Finland.

18 Institute for Nuclear Research and Nuclear Energy, BG-1784 Sofia, Bulgaria.

19 INAF/Osservatorio Astronomico and INFN Trieste, I-34131 Trieste, Italy.

20 Università di Pisa and INFN Pisa, I-56126 Pisa, Italy.

21 ICREA and Institut de Cienciès de l'Espai, IEEC-CSIC, E-08193 Bellaterra,
} Spain. 
turned out to be the strongest source of steady VHE $\gamma$-ray emission in the Galaxy. It is therefore used as the standard "calibration candle" for ground-based $\gamma$-ray experiments. Apart from testing the performance of $\gamma$-ray instruments, another aim of measuring the Crab Nebula is to increase the measurement precision of the Crab Nebula flux and the energy range covered. These continuous efforts provide insights necessary for the understanding of the very details of the emission mechanisms of VHE $\gamma$-rays in the Crab Nebula and pulsar. Important questions remain to be answered concerning the emission mechanisms of the nebula, of the pulsar at GeV energies, and of the nebula around PeV energies. Since its discovery, detailed studies of the VHE emission energy spectrum, ranging from several hundred $\mathrm{GeV}$ up to $80 \mathrm{TeV}$, have been carried out (e.g., Akerlof et al. 1990; Vacanti et al. 1991; Baillon et al. 1991; Goret et al. 1993; Konopelko et al. 1996; Tanimori et al. 1998; Hillas et al. 1998; Amenomori et al. 1999; Majumdar et al. 2002; Aharonian et al. 2004, 2006). Between 10 and $\sim 200 \mathrm{GeV}$, observations are sparse. A few results are provided by converted solar concentrator arrays that use the wave front sampling technique (Oser et al. 2001; de Naurois et al. 2002; Arqueros et al. 2002; Smith et al. 2006). However, the wave front sampling suffers from relatively large uncertainties in the calculation of the effective area and of the energy, as well as from poor $\gamma /$ hadron discrimination, which make it difficult to perform differential flux measurements.

A good explanation of the nebular dynamics and the observed energy spectrum below GeV energies can be obtained with the magnetohydrodynamic model suggested first by Rees \& Gunn (1974) and developed further by Kennel \& Coroniti (1984a, $1984 b)$. In this framework, the pulsar provides a continuous flow of charged particles (pulsar wind) with Lorentz factors of $10^{6}-10^{7}$. A standing reverse shock forms where the wind ram pressure balances the total pressure of the nebula. Wind particles accelerate in the shock to ultrarelativistic energies and subsequently lose their energy by synchrotron emission. The presence of synchrotron emission up to a few hundred $\mathrm{MeV}$ in conjunction with the observed $\gamma$-ray spectrum at TeV energies shows that particle acceleration takes place up to energies of $\sim 10^{15}-10^{16} \mathrm{eV}$. From the total luminosity of the synchrotron emission, it can be inferred that about $10 \%$ of the pulsar's energy loss rate is converted into the kinetic energy of particles.

Above $1 \mathrm{GeV}$ the dominant source of $\gamma$-ray emission is most likely inverse Compton (IC) scattering of synchrotron photons by the synchrotron-emitting electrons in the shocked wind region (synchrotron self-Compton model [SSC]; Gould 1965; Weekes et al. 1989; de Jager \& Harding 1992). To explain the observed VHE $\gamma$-ray spectrum, several other seed photon fields are also believed to contribute to the inverse Compton scattering, namely, far-infrared excess, cosmic microwave background, and millimeter photons (e.g., Aharonian et al. 2004).

Although the IC mechanism gives a good description of the observed energy spectrum between $500 \mathrm{GeV}$ and about $10 \mathrm{TeV}$, other processes may contribute in part to the VHE $\gamma$-ray emission. It is likely that a significant fraction of the mechanical energy lost by the pulsar is taken away by a hadronic component in the wind. Following interactions of this component with the interstellar medium, VHE $\gamma$-rays are emitted by decaying $\pi^{0}$ 's, which modify the energy spectrum at TeV energies and beyond (Atoyan \& Aharonian 1996; Bednarek \& Protheroe 1997; Bednarek \& Bartosik 2003; Amato et al. 2003). Atoyan \& Aharonian (1996) discuss the possibility of an "amplified" bremsstrahlung flux at GeV energies, which could account for the discrepancy between the measured $\mathrm{GeV} \gamma$-ray flux and predictions within the SSC framework (de Jager et al. 1996). If this is true, one should observe, in good approximation, a power-law spectrum between $100 \mathrm{GeV}$ and $10 \mathrm{TeV}$ with a spectral index 2.5-2.7. Another mechanism to be mentioned is IC scattering of relativistic electrons in the unshocked pulsar wind. If the target photons are emitted by the pulsar, a pulsed component could extend to $\gamma$-ray energies of several $100 \mathrm{GeV}$ (Bogovalov \& Aharonian 2000). An independent measurement in the intervening region between 60 and $400 \mathrm{GeV}$ would constrain further the parameters of various models. The MAGIC imaging atmospheric Cerenkov telescope (IACT) has a low-energy trigger threshold $(\sim 50 \mathrm{GeV})$ and is currently the only experiment capable of exploring this energy regime.

The spatially resolved morphology of the nebula is of a complex nature. Its size in optical bandwidths is about $4^{\prime} \times 6^{\prime}$. Due to synchrotron losses, the high-energy electrons will have shorter cooling times and only the lower energy electrons will reach out farther into the nebula. Thus, the effective source size is expected to shrink with increasing energy of the radiation. The radio emission is expected to extend up to and beyond the filaments optically visible, whereas X-ray and multi-TeV $\gamma$-rays should be produced in the vicinity of the shock. On the other hand, the expected source size would increase if the presence of an ionic component is established. In a special study the HEGRA collaboration concluded that the rms size of the VHE $\gamma$-ray emission region is $<1.5^{\prime}$ for energies above $1 \mathrm{TeV}$ (Aharonian et al. 2000). A similar study at energies below $1 \mathrm{TeV}$ has not been performed up to now.

The Crab pulsar is a source of pulsed radiation and has been detected up to $\mathrm{GeV}$ energies. The Crab pulsar has a period of $33 \mathrm{~ms}$ and was first discovered in radio (Staelin \& Reifenstein 1968) and shortly afterward as the first pulsar in the optical domain (Cocke et al. 1969). Since then, pulsed emission from the Crab pulsar has been detected at all accessible energies up to $\gamma$-rays (for a compilation of the broadband emission see Thompson et al. 1999). EGRET detected the Crab pulsar in $\gamma$-rays up to energies of $10 \mathrm{GeV}$ with a hint of a cutoff in the energy spectrum in the highest energy bin at $6 \mathrm{GeV}$ (Nolan et al. 1993). Despite various efforts, observations from the ground at higher energies have, so far, failed to detect pulsed emission (e.g., Musquere 1999; Aharonian et al. 1999, 2004; Lessard et al. 2000; de Naurois et al. 2002). Some experiments reported episodic pulsed emission (Gibson et al. 1982; Bhat et al. 1986; Acharya et al. 1992) and persistent pulsed emission over a $1 \mathrm{yr}$ period (Downthwaite et al. 1984), but these observations have not been confirmed by other experiments.

The high-energy emission from the pulsar is assumed to be due to curvature and synchrotron radiation from relativistic charged particles that are forced to move along magnetic field lines inside the magnetosphere of the pulsar. The question of where the particles are being accelerated is the subject of ongoing theoretical activities. In the two most popular models, the production of electrons and positrons and their acceleration take place either above the polar cap of the neutron star (e.g., Harding et al. 1978; Daugherty \& Harding 1982) or in outer gaps in between the null surface and the light cylinder of the magnetosphere (e.g., Cheng et al. 1986a, 1986b; Chiang \& Romani 1992). We should not omit the slot gap model, which places the acceleration zone at the outer rim of the polar cap (Arons 1983; Muslimov \& Harding 2003). These models differ in the predicted shape and cutoff of the energy spectrum at the highest energies. A measurement of the turnover in the spectrum would shed light on the possible sites of the particle acceleration and can constrain models.

In this paper we report about the observation of the Crab Nebula and pulsar with the MAGIC telescope between 2005 
October and December. After describing the MAGIC telescope and the performed observations in $\S \S 2$ and 3, we present the analysis chain in $\S 4$. In $\S 5$ we present our results on the steady emission comprising the differential flux between $60 \mathrm{GeV}$ and $9 \mathrm{TeV}$, a study of the morphology of the emission region, and the measured integral flux above $150 \mathrm{GeV}$. In $\S 5.4$ we present the results of our search for pulsed emission, and we close with a discussion of our results in $\S 6$.

\section{THE MAGIC TELESCOPE}

The MAGIC (Major Atmospheric Gamma Imaging Cerenkov) telescope (see Lorenz 2004) is located on the Canary Island, La Palma (2200 $\mathrm{m}$ asl, $28.45^{\circ}$ north, $17.54^{\circ}$ west). MAGIC is currently the largest single-dish IACT. It has a $17 \mathrm{~m}$ diameter tessellated reflector consisting of $9640.5 \times 0.5 \mathrm{~m}^{2}$ diamondmilled aluminum mirrors, which are grouped onto support panels in units of four. Depending on the elevation of the telescope, the position of every panel is adjusted by computer-controlled actuators of the so-called Active Mirror Control, thus providing optimal focusing. About $80 \%$ of the light from a point source is focused within a radius of $0.05^{\circ}$ in the focal plane. The MAGIC telescope is focused to a distance of $10 \mathrm{~km}$, the most likely location of the shower maximum for $50 \mathrm{GeV} \gamma$-ray air showers at small zenith angles.

The faint Cerenkov light flashes produced in air showers are recorded by a camera comprising 577 photomultiplier tubes (PMTs). The inner part of the camera (radius $\sim 1.1^{\circ}$ ) is equipped with 397 PMTs (type ET 9116A; 1" diameter from Electron Tubes Ltd [ET]) with a diameter of $0.1^{\circ}$ each. The outer part of the camera is equipped with 180 PMTs (type ET 9117A; 1.5" diameter) with a diameter of $0.2^{\circ}$. The central PMT is modified for optical pulsar studies (Lucarelli et al. 2005). Hollow hexagonal nonimaging light concentrators (often called light catchers) are placed in front of all photomultipliers to compensate for the dead space between them and in order to shrink the observation solid angle to the reflector. The entrance window of the PMTs is coated with a diffuse lacquer doped with a wavelength shifter (WLS; Paneque et al. 2003). The combination of the hemispherically shaped PMT, the light catcher, the diffuse coating, and the WLS results in a $15 \%-25 \%$ higher quantum efficiency compared to flat window PMTs. For protection purposes (humidity, dust) a thin entrance window made of Plexiglas (type UG-218, with a UV cutoff around $290 \mathrm{~nm}$ ) is placed in front of the camera.

The PMTs have 6 dynodes and operate at a gain of roughly 30,000 to slow down aging and damage from high currents by light during observations close to the Galactic plane and during moonshine. After amplification, the fast analog signals are converted to optical signals and transported by $160 \mathrm{~m}$ long optical fibers to the counting house. There the optical signal is converted back and split. Part of the signal is routed to the trigger. The current configuration of the MAGIC camera has a trigger region of $2.0^{\circ}$ in diameter (Cortina et al. 2005). This provides a $\gamma$-ray trigger collection area of the order of $10^{5} \mathrm{~m}^{2}$ (at $200 \mathrm{GeV}$ for a source close to zenith). Presently, the trigger energy range spans from $50-60 \mathrm{GeV}$ (peak in the differential trigger rates at small zenith angles for a $\gamma$-ray source with a spectral slope of -2.6 ) up to tens of TeV. An event is triggered if the signals in each of 4 neighboring pixels exceed a threshold of $\sim 7$ photoelectrons (phe) within a coincidence time window of 6 ns.

Before being digitized by an 8 bit, 300 MSamples/s FADC system, each signal is stretched to an FWHM of about 6 ns. The FADC continuously writes the digitized amplitude information into a ring buffer. In case of a trigger the digitization stops and the corresponding part of the ring buffer is written onto a disk.
The dead time introduced by the readout is $25 \mu \mathrm{s}$. In order to expand the dynamic range to $\sim 1000$, the signal of every PMT is split into two branches, differing by a factor of 10 in gain. The higher gain branch is read out for a $50 \mathrm{~ns}$ time interval. When the signal amplitude exceeds a preset threshold, the delayed lower gain is routed to the same FADC channel and recorded in the following $50 \mathrm{~ns}$. Otherwise, the signal from the high gain branch continues to be recorded and is used to determine the pedestal offset of each PMT channel.

The accuracy in reconstructing the direction of incoming $\gamma$-rays on an event-by-event basis, hereafter $\gamma$-ray point-spread function or $\gamma$-PSF, is about $0.1^{\circ}$, depending on the energy. With the information provided by a starguider camera, mispointing is corrected to an absolute precision of about $1^{\prime}$. A $\gamma$-ray source with an absolute intensity of $\sim 2 \%$ of the Crab Nebula and similar spectrum can be detected with MAGIC within $50 \mathrm{hr}$ at energies $>200 \mathrm{GeV}$ on a significance level of $5 \sigma$.

\section{OBSERVATIONS AND DATA SELECTION}

Observations of the Crab Nebula with MAGIC are conducted on a regular basis, as a means to monitor the performance of the telescope. In this report we restrict ourselves to the analysis of data obtained in the first observation cycle of the MAGIC telescope between 2005 October and December. The observations were performed in the so-called ON/OFF mode. The telescope was pointed toward the Crab pulsar (ON) for about $16 \mathrm{hr}$. An OFF source position, a sky region where no $\gamma$-ray source is known, was observed in the same range of zenith angles as the ON source. For the background estimation we used OFF data collected for over $19 \mathrm{hr}$.

One of the main objectives of this analysis was to explore the lower energy range of accessible $\gamma$-ray energies. The energy threshold of IACTs depends strongly on the zenith angle of observation. Restriction to events with low zenith angles provides the lowest possible energy threshold. Therefore, we select events with zenith angles $\$ 20^{\circ}$. For any given night the data affected by technical problems or fluctuations in the data rate in excess of $10 \%$ were rejected. The atmospheric conditions were judged from the nightly averaged and publicly available atmospheric extinction coefficients from the nearby Carlsberg Meridian telescope. ${ }^{22}$ Within the selected nights, the atmospheric light transmission changed by less than $5 \%$. The nights with data that survived all the selection criteria, together with the corresponding observation times, trigger rates, and zenith angle range, are listed in Table 1. The selected data sample comprises 14 nights amounting to a total ON observation time of 955 minutes ( $\sim 16 \mathrm{hr})$.

\section{DATA ANALYSIS}

The data analysis was carried out using the standard MAGIC analysis and reconstruction software MARS (Bretz \& Wagner 2003). After removing faulty and unstable camera channels, which amount to $3 \%-5 \%$ of the total number of PMTs, the signal amplitudes, extracted with the digital filtering method (Albert et al. 2006), were converted to phe by using the F-factor method (Mirzoyan \& Lorenz 1997). Using calibration events recorded interlaced to normal events (Gaug et al. 2005), the conversion factors were updated every $10 \mathrm{~s}$. There is a $10 \%$ systematic uncertainty in the calibration that directly propagates to the uncertainty of the event energy scale (point 7 in Table 4 below). Time offsets between pixels are corrected with a precision of better than $1 \mathrm{~ns}$.

${ }^{22}$ See http://www.ast.cam.ac.uk/ dwe/SRF/camc_extinction.html. 
TABLE 1

Data Selected for Analysis

\begin{tabular}{|c|c|c|c|}
\hline $\begin{array}{c}\text { Date } \\
\text { (MJD) }\end{array}$ & $\begin{array}{c}\text { Rates } \\
(\mathrm{Hz})\end{array}$ & $\begin{array}{l}\text { On Time } \\
\text { (minutes) }\end{array}$ & $\begin{array}{l}\text { Zd Range } \\
\text { (deg) }\end{array}$ \\
\hline 53,648 .. & 130 & 73 & $7-23$ \\
\hline $53,655 \ldots \ldots$ & 115 & 100 & $7-19$ \\
\hline $53,671 \ldots \ldots \ldots \ldots \ldots \ldots \ldots \ldots \ldots \ldots \ldots$ & 122 & 105 & $7-23$ \\
\hline $53,672 \ldots$ & 105 & 61 & $8-20$ \\
\hline $53,679 \ldots \ldots \ldots \ldots \ldots \ldots \ldots \ldots \ldots \ldots \ldots \ldots$ & 115 & 51 & $7-20$ \\
\hline $53,684 \ldots \ldots \ldots \ldots \ldots \ldots \ldots \ldots \ldots \ldots \ldots \ldots$ & 95 & 50 & $7-20$ \\
\hline $53,707 \ldots \ldots \ldots \ldots \ldots \ldots \ldots \ldots \ldots \ldots \ldots \ldots$ & 98 & 53 & $7-11$ \\
\hline $53,709 \ldots$ & 105 & 48 & $7-10$ \\
\hline $53,711 \ldots \ldots \ldots \ldots \ldots \ldots \ldots \ldots \ldots \ldots \ldots \ldots$ & 105 & 48 & $7-10$ \\
\hline $53,713 \ldots \ldots \ldots \ldots \ldots \ldots \ldots \ldots \ldots \ldots \ldots$ & 108 & 50 & $7-14$ \\
\hline $53,727 \ldots \ldots \ldots \ldots \ldots \ldots \ldots \ldots \ldots \ldots \ldots$ & 92 & 44 & $7-13$ \\
\hline $53,729 \ldots \ldots \ldots \ldots \ldots \ldots \ldots \ldots \ldots \ldots$ & 97 & 61 & $7-13$ \\
\hline $53,731 \ldots \ldots \ldots \ldots \ldots \ldots \ldots \ldots \ldots \ldots \ldots$ & 100 & 107 & $7-14$ \\
\hline $53,735 \ldots \ldots \ldots \ldots \ldots \ldots \ldots \ldots \ldots \ldots \ldots$ & 92 & 104 & $7-15$ \\
\hline
\end{tabular}

NoтE.-The second column lists the average event rate after tail cuts and a cut in SIZE $>100$ phe (for the definition of SIZE see text).

After calibrating the data, pixels with faulty reconstructed signal amplitudes and times were rejected and the corresponding amplitude and time information was interpolated from the signals and times of neighboring pixels. Before image parameterization, a tail-cut cleaning of the image was performed, requiring signals higher than a predefined absolute amplitude level and time coincidences (3.3 ns) with neighboring channels. The time coincidence effectively suppresses pixels containing only a signal from the night sky. For most of the analysis the minimum required pixel content is 6 phe for so-called core pixels and 4 phe for boundary pixels. For the morphology studies the minimum pixel contents were raised to 10 phe (core) and 8 phe (boundary), respectively, which improves the angular resolution, albeit at the expense of an increased analysis threshold.

Every cleaned event was parameterized by a principal component analysis, commonly referred to as Hillas parameterization (Hillas 1985). The parameterization was later used to separate between $\gamma$-ray event candidates and background event candidates. The Hillas parameters DIST, LENGTH, WIDTH, and also

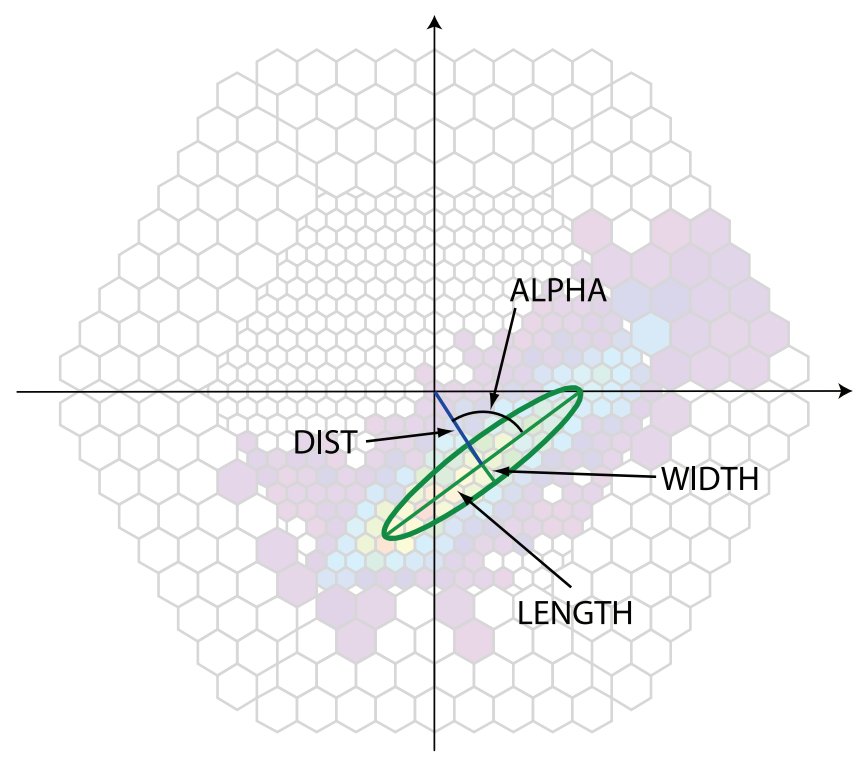

Fig. 1.-Parameterization of a shower image with Hillas parameters.

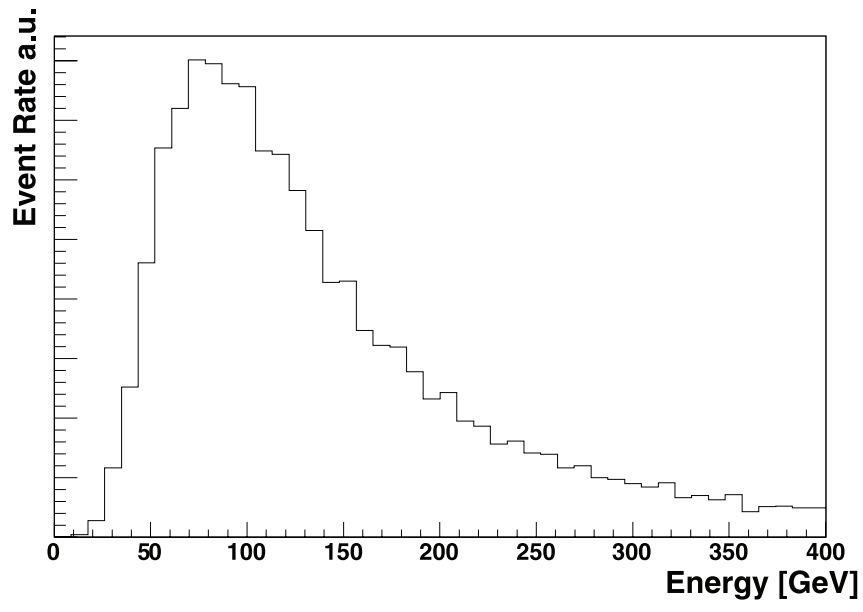

FIG. 2.-Energy distribution of MC $\gamma$-ray events with SIZE $>100$ phe for a simulated $\gamma$-ray source with spectral slope -2.6 .

ALPHA are illustrated for a recorded shower image in Figure 1. Another useful parameter is the SIZE of a shower image, the intensity of the image after image cleaning in units of recorded photoelectrons. Note that SIZE depends on the applied tail cuts. SIZE is a good estimate of the primary particle energy, provided that the shower impact distance to the telescope principal axis is below $\sim 120 \mathrm{~m}$. An event preselection was performed by discarding event candidates affected by noise and pickup (e.g., car flashes) and event candidates with a low number of pixels (after tail cuts typically a minimum number of 5 core pixels were requested). In addition, an image SIZE of at least $\sim 100$ phe was requested. Figure 2 shows the energy distribution of simulated $\gamma$-ray events with SIZE $>100$ phe. The distribution peaks at an energy of $75 \mathrm{GeV}$. The simulated $\gamma$-ray source has a power-law spectrum with an index of -2.6 .

A Monte Carlo (MC) simulation properly describing data is a necessary requisite for a ground-based $\gamma$-ray experiment. In Figure 3 the image parameter WIDTH is shown for simulated $\gamma$-rays and $\gamma$-rays extracted from data. The four panels are for consecutive bins in SIZE covering the entire range of analyzed $\gamma$-ray energies. For an unbiased comparison, loose cuts have been applied in the $\gamma /$ hadron separation (explained in the next section). The $\gamma$-ray excess was obtained by subtracting the scaled distribution of the OFF-data sample from the distribution of the ON-data sample. The scaling factor was found by normalizing the $\mid$ ALPHA $\mid$ distributions of both samples between $\mid$ ALPHA $\mid=$ $30^{\circ}$ and $70^{\circ}$. The comparison was done by selecting events with small $\mid$ ALPHA $\mid$ (typically less than $10^{\circ}$ ). A small $\mid$ ALPHA $\mid$ is expected for $\gamma$-rays from the Crab Nebula, as explained later. The agreement of the MC simulated distributions and the distributions extracted from data is acceptable in all four SIZE bins. However, it is evident from the figures that the agreement worsens at large SIZE. In the analysis, a possible bias introduced by this behavior is avoided by applying rather loose cuts at energies above several $\mathrm{TeV}$.

\subsection{Gamma Hadron Separation}

Only a small fraction between $10^{-3}$ and $10^{-4}$ of the recorded data are $\gamma$-ray showers. The major fraction of the recorded events are cosmic rays of hadronic origin. This unwanted background has to be suppressed offline. For this purpose we applied a multivariate method, the random forest (Breiman 2001; Bock et al. 2004; Albert et al. 2008), which uses the image parameters to compute the HADRONNESS of an event. The HADRONNESS 

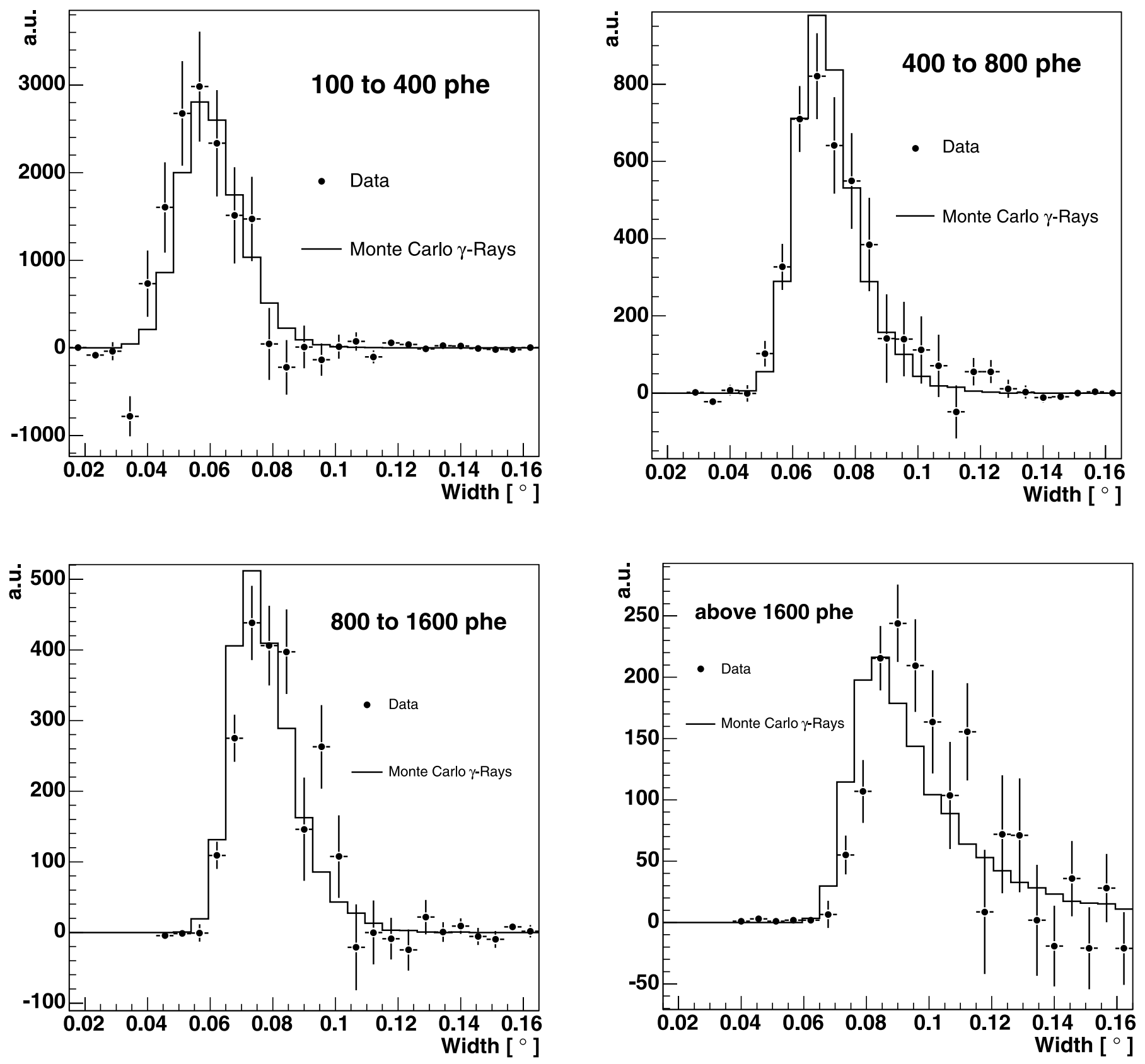

FIG. 3.-Distribution of the image parameter WIDTH for $\gamma$-ray events extracted from data and for MC simulated $\gamma$-rays in four consecutive bins of SIZE covering the full analyzed energy range.

of an event quantifies the probability of an event to be " $\gamma$-like" or "hadron-like." The random forest is trained with MC simulated $\gamma$-ray events and either with simulated hadronic cosmic-ray events or, as in the present case, with background events recorded by MAGIC. In this study, we used for the training of the random forest the image parameters SIZE, DIST, WIDTH, and LENGTH, as well as the third moment along the major axis of a shower image and a parameter describing the CONCENTRATION of a shower image.

Figure 4 shows the HADRONNESS for MC simulated $\gamma$-ray showers (red) and for recorded background (blue) as a function of SIZE. A clear separation between both populations is visible for SIZE $\gtrsim 300$ phe, corresponding to $\gamma$-ray energies $\gtrsim 150 \mathrm{GeV}$. Below 300 phe both populations start to overlap until, at $\sim 200$ phe $(\sim 100 \mathrm{GeV})$, no more separation is possible.
The bottom panel in the figure shows the maximum quality factor $Q\left(\epsilon_{\gamma} / \epsilon_{B}^{1 / 2}\right)$ for each SIZE interval obtained for an optimized HADRONNESS cut. Parameter $\epsilon_{\gamma}$ is the fraction of retained $\gamma$-ray events and $\epsilon_{B}$ the fraction of retained background events. The corresponding HADRONNESS cut is shown by the stars in the top panel of the figure.

The random forest method is also used to estimate the energy of each event (Albert et al. 2008). An energy resolution of $\sim 25 \%$ is achieved for events with energies $>200 \mathrm{GeV}$. The energy resolution reduces to $\sim 40 \%$ around $70 \mathrm{GeV}$.

\subsection{Signal Extraction}

After $\gamma /$ hadron separation and energy estimation, the $\gamma$-ray signal is extracted from an $\mid$ ALPHA $\mid$ distribution. ALPHA is the angle between the major axis of the recorded shower and the 

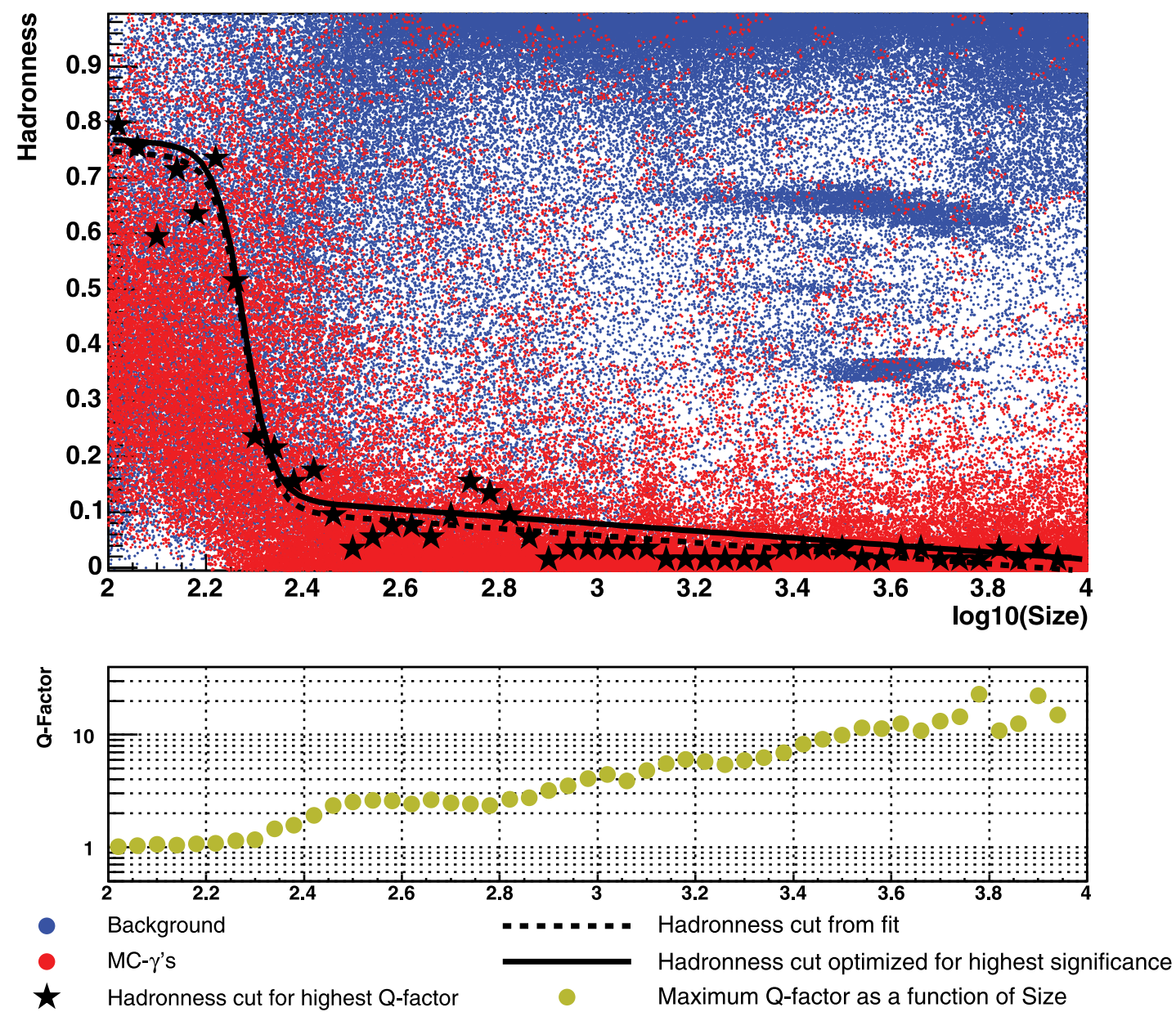

FIG. 4.-Top: Distribution of the parameter HADRONNESS vs. SIZE. The background events are marked blue and the MC $\gamma$-ray events red. Note that below a SIZE of 300 phe most of the background events are hidden behind the simulated $\gamma$-ray events. For bins in SIZE the HADRONNESS cuts that yield the highest quality factors (black stars) are found. The dashed line is a fit to the HADRONNESS cut values. The solid line is the fit shifted by a constant value and results in the $\gamma$-ray signal from the Crab Nebula with the highest significance. Bottom: Quality factor for the HADRONNESS cut (stars) in the top panel.

vector connecting its center of gravity $(\mathrm{CoG})$ with the source position in the camera plane (cf. Fig. 1). Shower images of $\gamma$-rays from the source point with their major axes toward the source position in the camera and appear as an excess at small values in the $\mid$ ALPHA $\mid$ distribution. Figure 5 shows two |ALPHA| distributions from the data (black: ON data; red: OFF data). The left panel in Figure 5 shows the $\mid$ ALPHA $\mid$ distribution of events with estimated energies between 80 and $100 \mathrm{GeV}$; the right panel shows the distribution of events with reconstructed energies above $200 \mathrm{GeV}$. In both cases an excess of $\gamma$-ray events is clearly visible. However, the significance of the $\gamma$-ray signal at lower energies is considerably reduced compared to higher energies because of the degradation of the background suppression toward lower energy (see Fig. 4).
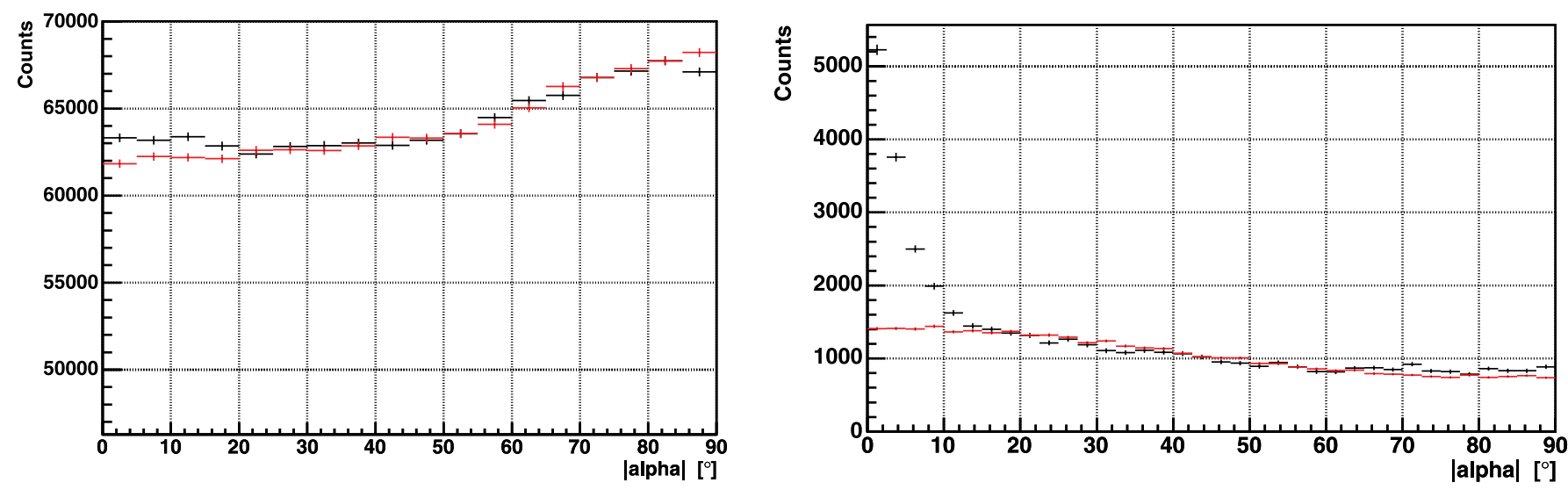

FIG. 5.-Distribution of the image parameter $|\mathrm{ALPHA}|$ for the bin of reconstructed energy $80-100 \mathrm{GeV}$ in the left panel and for events with energies $>200 \mathrm{GeV}$ in the right panel. (black: ON data; red: OFF data) 


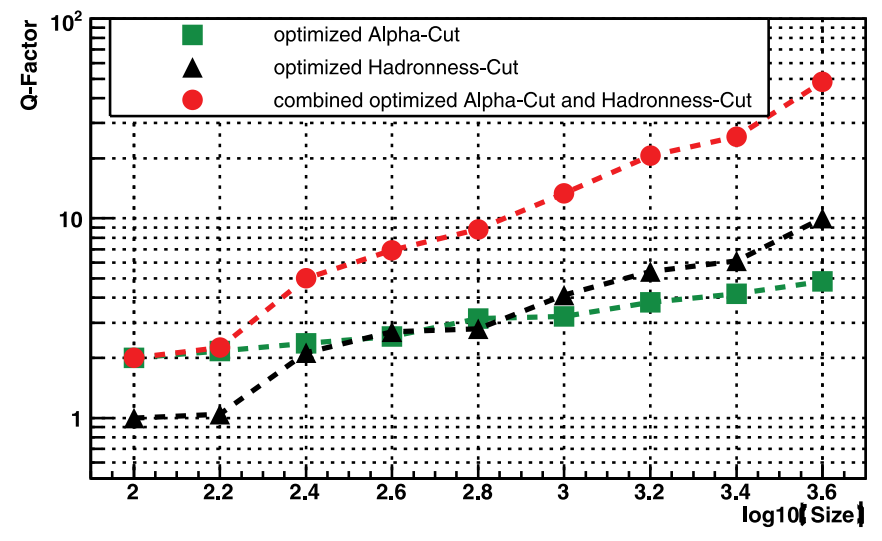

FIG. 6.- Size dependence of the $Q$-factor for optimized $\mid$ ALPHA $\mid$ cut and HADRONNESS cut, and the $Q$-factor for the combination of $\mid$ ALPHA $\mid$ cut and HADRONNESS cut.

At the lowest energies ALPHA is currently the only means by which it is possible to separate $\gamma$-rays and background events. This is illustrated in Figure 6, which shows the quality factor as a function of SIZE separate for an optimized ALPHA cut and optimized HADRONNESS cut, as well as the combination of both cuts. Below SIZE 250 phe a cut in HADRONNESS does not improve $\gamma /$ hadron separation and reduces only statistics. On the other hand, with ALPHA a quality factor of 2 is still possible.

\subsection{Sensitivity}

The highest integral sensitivity for $\gamma$-ray emission from the Crab Nebula is obtained above $\sim 250 \mathrm{GeV}$. The integral sensitivities for energies above $250 \mathrm{GeV}$ obtained on a daily basis are listed in Table 2. The sensitivities are calculated in units of $\sigma_{\mathrm{LiMa}} /(\text { hours })^{1 / 2}$; the significance $\sigma_{\mathrm{LiMa}}$ is calculated using equation (17) from $\mathrm{Li}$ \& Ma (1983). In the last column of the table, the sensitivity is expressed as the minimum flux, normalized to the Crab flux that can be measured with $5 \sigma$ significance ${ }^{23}$ in a $50 \mathrm{hr}$ observation. The day-by-day sensitivities vary by about $10 \%$, indicating a stable telescope performance throughout the observations.

The energy dependence of the sensitivity was studied by calculating the integral sensitivity for several analysis thresholds. The SIZE-dependent HADRONNESS cut (Fig. 4, black solid line) was used for $\gamma /$ hadron separation, and the ALPHA cut was tightened with increasing energy based on studies from MC simulations. The integral sensitivity for a $50 \mathrm{hr}$ observation is $13 \%$ $\mathrm{Crab}$ at $75 \mathrm{GeV}$ and improves continuously with increasing the analysis threshold to about $2.2 \%$ above $\sim 250 \mathrm{GeV}$ (see Table 3 ). Note that above $1.6 \mathrm{TeV}$ the background is estimated from only two events, which results in an uncertainty of more than $70 \%$ on the integral sensitivity.

\subsection{Systematic Uncertainties}

Apart from statistical errors, many results in cosmic-ray experiments are affected by rather large systematic errors. One of the main problems is the lack of test beams that allow calibration of the entire instrument in combination with the showering process in the atmosphere. The standard replacement for a test beam calibration is the use of MC simulation based on many linked processes either using physical models (example: the simulation of electromagnetic showers) or calibrating them in separate measurements (example: the spectral mirror reflectivity). In some cases

\footnotetext{
${ }^{23}$ Note that in this case the significance is calculated as excess/(background $)^{1 / 2}$.
}

TABLE 2

MAGIC Integral Sensitivities to $\gamma$-Ray Emission from the Crab Nebula for $\gamma$-Ray Energies above $250 \mathrm{GeV}$

\begin{tabular}{|c|c|c|}
\hline $\begin{array}{c}\text { Date } \\
\text { (MJD) }\end{array}$ & $\begin{array}{c}\text { Sensitivity in } \\
{\left[\sigma_{\text {LiMa }} /\left(\text { hours }^{1 / 2}\right]\right.}\end{array}$ & $\begin{array}{c}\text { Sensitivity in } \\
(\% \mathrm{Crab}, 50 \mathrm{hr}, 5 \sigma)\end{array}$ \\
\hline $53,648 \ldots$ & 18.1 & 2.4 \\
\hline $53,655 \ldots \ldots$ & 19.6 & 2.1 \\
\hline $53,671 \ldots \ldots \ldots \ldots \ldots \ldots \ldots \ldots \ldots$ & 19.9 & 2.1 \\
\hline $53,672 \ldots$ & 19.1 & 2.2 \\
\hline 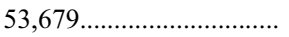 & 19.4 & 2.2 \\
\hline $53,684 \ldots \ldots$ & 21.1 & 1.8 \\
\hline 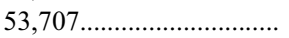 & 23.6 & 2.0 \\
\hline $53,709 \ldots$ & 16.1 & 2.7 \\
\hline $53,711 \ldots \ldots$ & 18.9 & 2.2 \\
\hline $53,713 \ldots \ldots \ldots$ & 18.7 & 2.2 \\
\hline $53,727 \ldots \ldots \ldots \ldots \ldots \ldots \ldots \ldots \ldots \ldots \ldots \ldots \ldots \ldots \ldots$ & 19.1 & 2.3 \\
\hline $53,729 \ldots \ldots \ldots \ldots \ldots \ldots \ldots \ldots \ldots \ldots \ldots \ldots \ldots \ldots$ & 17.5 & 2.3 \\
\hline 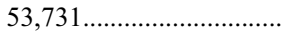 & 18.0 & 2.3 \\
\hline $53,735 \ldots \ldots \ldots \ldots \ldots \ldots \ldots \ldots \ldots \ldots \ldots \ldots \ldots \ldots$ & 18.7 & 2.1 \\
\hline Average ........................ & $19.1 \pm 0.5(1.7)$ & $2.21 \pm 0.05(0.2)$ \\
\hline
\end{tabular}

Notes.-Average values of each column are given in the last row with the corresponding rms value in parentheses. Note that different definitions of significance are used in the two columns.

one has to make reasonable guesses (example: photoelectron collection efficiency in the PMT front-end volume). The calibration of individual effects suffers partly from cross-correlations, which are not always well understood. Currently, the best approach is to estimate the systematic uncertainties (commonly called systematic errors) of the various parameters separately and to combine them to a global systematic error. Here we follow the general practice of adding these individual errors in quadrature although this will result in a slight underestimate of the total systematic error. Table 4 lists the dominant systematic error contributions $(\geq 2 \%)$ for the spectral parameters (flux, slopes, cutoffs, etc.). The systematic errors influence the spectrum in different ways. Some $(\mathrm{Nr} 1-7,10,11)$ result in an uncertainty of the energy scale and thus can enter as a large factor in the flux at a given energy in case of steep spectra with slopes $<3$; others $(\mathrm{Nr} 8,9,12-13,15-17)$ linearly influence the flux normalization for the spectrum.

The most critical contributions to the systematic error come from the uncertainties in the conversion of photons to measurable photoelectrons (combined under item 7), the so-called photon detection efficiency (PDE). The PDE is a combination of many small effects such as the reflectivity variation of the light catchers, tolerances in the light catcher geometry, angular effects on the PMT surface, nonuniformity of the diffuse lacquer coating, the QE spread and cathode nonuniformity of the PMTs, the photoelectron collection efficiency in the PMT front-end volume, and gain variations of the first dynode. Also some contribution of the signal transmission to the DAQ is included.

Fortunately, the PDE can normally be measured with a light source uniformly illuminating the camera with short blue or UV light pulses. Obviously, the light pulser itself introduces some systematic errors such as in the absolute light flux determination, small deviations from uniformity in the illumination, some (small) temperature drift, and amplitude jitter. Also, the used method of determining the number of detected photoelectrons, the above-mentioned so-called F-factor method, introduces some uncertainty.

Another rather big uncertainty is the effective reflectivity of the mirrors defined as the light from a source at infinity being 
TABLE 3

Integral Sensitivities of the MAGIC Telescope to the $\gamma$-Ray Emission from the Crab Nebula for Several Analysis Thresholds

\begin{tabular}{|c|c|c|c|c|c|}
\hline $\begin{array}{l}\text { Energy } \\
(\mathrm{GeV})\end{array}$ & $\begin{array}{c}\mid \text { ALPHA } \mid \text { Cut } \\
\text { (deg) }\end{array}$ & ON Events & OFF Events & Excess Events & $\begin{array}{c}\text { Sensitivity } \\
(\% \text { Crab, } 50 \mathrm{hr}, 5 \sigma)\end{array}$ \\
\hline$>75 \ldots \ldots$ & $<10$ & 232505 & 221751 & 10754 & 13.0 \\
\hline$>110$ & $<10$ & 58600 & 49702 & 8898 & 7.5 \\
\hline$>200$ & $<10$ & 11399 & 4960 & 6439 & 3.3 \\
\hline$>400$ & $<7.5$ & 2866 & 348 & 2518 & 2.2 \\
\hline$>800$ & $<5$ & 613 & 20 & 593 & 2.3 \\
\hline$>1600$ & $<5$ & 43 & 2 & 41 & 1.0 \\
\hline
\end{tabular}

focused onto the area of a pixel. Comparing the measured brightness of a star and its image back-reflected by a high-quality diffuse reflector in the camera plane allows one to carry out a routine reflectivity measurement (see Fig. 7), with an uncertainty of about $7 \%$. A similarly large error contribution was estimated for the event reconstruction. Again, many small effects contribute to the reconstruction losses or to the wrong assignment of events. In contrast to the procedure to limit the uncertainty as in the example of the PDE, no simple method to cross-check the error range of the reconstruction efficiency is possible and a reasonable guess had to be made.

Effects that influence the slope of reconstructed $\gamma$-ray energy spectra (class $\mathrm{C}$ effects in Table 4) are mostly dominating at the lowest and highest accessible energies. The estimate of the systematic slope error is rather difficult. In case of a power law or moderately curved power law we estimate an uncertainty on the slope of 0.2 . We note that measurements by current secondgeneration telescopes of the spectral slope of the Crab Nebula agree better than 0.1 in the overlapping energy range.

In summary, we obtain a systematic energy scale error of $16 \%$, a systematic error of $11 \%$ on the flux normalization (without the energy scale error), and a systematic slope error of \pm 0.2 (which is a combination of error 13 and the other relevant class A errors averaged over the energy).

\section{ANALYSIS RESULTS}

\subsection{Differential Energy Spectrum of the Crab Nebula}

By extracting the $\gamma$-ray signal in each bin of the reconstructed energy $E_{\text {rec }}$, a spectrum $N_{i}$ of $\gamma$-rays in each $E_{\text {rec }}$ bin $i$ can be constructed. The reconstructed energy is subject to a bias. Before determining a differential $\gamma$-ray flux in true energy $E_{\text {true }}$ bins, the spectrum $N_{i}$ has, therefore, to be converted into a spectrum $M_{j}$ of $\gamma$-rays in bins of $E_{\text {true }}$. This is done by applying an unfolding procedure with regularization (Anykeyev et al. 1991). An essential input for the unfolding procedure is the migration matrix, which describes the migration of events from bin $i$ in $E_{\text {rec }}$ into a bin $j$ of $E_{\text {true }}$. The migration matrix is determined from MC simulated $\gamma$-ray showers. The unfolding is done independently for different regularization schemes (Tikhonov \& Arsenin 1979; Bertero 1989; Schmelling 1994; Albert et al. 2007b). Figure 8 shows one distribution of excess events from the Crab Nebula after unfolding by the method of Bertero (1989). The integral rate of excess events is $0.4 \mathrm{~Hz}$. The differences between the unfolded points $M_{j}$ obtained with the different regularization schemes are used to estimate a systematic error due to the unfolding. Figure 10 shows the differential $\gamma$-ray flux, which was obtained with the regularization scheme proposed by Bertero (1989) and by normalizing the unfolded spectrum $M_{j}$, to the effective collection area (Fig. 9), the effective observation time, and the bin width of $E_{\text {true }}$ (given by the horizontal bars at each flux point in the figure). The average differential flux for each energy bin is presented in Table 5.

The influence of different choices for tail cuts, HADRONNESS cuts, DIST cuts, and core pixel cuts on the measured flux is indicated in Figure 10 by the shaded region and quoted as systematic uncertainty in Table 5. Due to analysis uncertainties, the band broadens at low energies, mostly because of limited $\gamma /$ hadron discrimination power. It broadens at the highest energies due to low event statistics.

The energy spectrum is parameterized with both a power-law and a curved power-law Ansatz. The fit takes into account correlations between the spectral points that are introduced by the unfolding procedure. A correlated fit with a power law

$$
\frac{d F}{d E}=f_{0}(E / 300 \mathrm{GeV})^{\Gamma}
$$

provides a flux normalization $f_{0}$ of $\left(5.7 \pm 0.2_{\text {stat }}\right) \times 10^{-10} \mathrm{~cm}^{-2}$ $\mathrm{s}^{-1} \mathrm{TeV}^{-1}$ and a spectral index $\Gamma$ of $-2.48 \pm 0.03_{\text {stat }} \pm 0.2_{\text {syst }}$. The $\chi^{2}$ of the fit is 24 for 8 degrees of freedom, which disfavors a pure power-law description of the spectrum. The energy spectrum is better described by a curved power-law Ansatz

$$
\frac{d F}{d E}=f_{0}(E / 300 \mathrm{GeV})^{\left[a+b \log _{10}(E / 300 \mathrm{GeV})\right]}
$$

yielding a flux normalization $f_{0}$ of $\left(6.0 \pm 0.2_{\text {stat }}\right) \times 10^{-10} \mathrm{~cm}^{-2} \mathrm{~s}^{-1}$ $\mathrm{TeV}^{-1}, a=-2.31 \pm 0.06_{\text {stat }}$, and $b=-0.26 \pm 0.07_{\text {stat }} \pm 0.2_{\text {syst }}$. The $\chi^{2}$ of the fit is 8 for 7 degrees of freedom.

Figure 11 shows the differential flux measurements multiplied by the energy squared, i.e., the spectral energy distribution (SED). In the figure we compare our measurement with those from other experiments. For energies above $400 \mathrm{GeV}$ the derived spectrum is in good agreement with measurements of other air Cerenkov telescopes (Hillas et al. 1998; Aharonian et al. 2004, 2006; Tanimori et al. 1998). At energies $<400 \mathrm{GeV}$, below the threshold of previous measurements by IACTs, we compare our results with those obtained by CELESTE (de Naurois et al. 2002; Smith et al. 2006) and STACEE (Oser et al. 2001), i.e., measurements performed by converted solar tower experiments. It should be noted that the integral flux values of these experiments had to be converted to differential ones by assuming a shape of the source spectrum, which causes an additional bias.

Above $1 \mathrm{TeV}$ up to about $10 \mathrm{TeV}$ the measured energy spectrum is well described by a pure power law (Weekes et al. 1989; Aharonian et al. 2004). Going to lower energies, one expects a continuous hardening of the spectrum. However, this could not be demonstrated by earlier measurements. The change of the 
TABLE 4

Contribution to the Systematic Uncertainties

\begin{tabular}{|c|c|c|c|c|}
\hline Item & Source of Uncertainty & Class & $\begin{array}{l}\text { Uncertainty } \\
\quad(\%)\end{array}$ & Comments \\
\hline 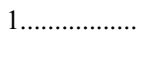 & Parametrization of atmosphere in $\mathrm{MC}$ simulation & A & 3 & $\begin{array}{l}\text { Deviations due to yearly and daily pressure changes, deviations of real density distribution } \\
\text { and standard atmosphere model }\end{array}$ \\
\hline $2 \ldots \ldots \ldots \ldots \ldots$ & Atmospheric transmission losses due to Mie scattering & $\mathrm{A},(\mathrm{C})$ & 5 & Lack of good measurements; short-term unpredictable changes possible \\
\hline 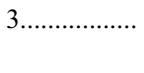 & Incorrect NSB simulation & A & 3 & $\begin{array}{l}\text { MC assumes uniform NSB; variations due to source location, air glow, variations } \\
\text { due to man-made light; stars in the FOV }\end{array}$ \\
\hline 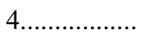 & Reflectivity of main mirror & A & 7 & From measurements of reflected star images \\
\hline $5 \ldots \ldots \ldots \ldots \ldots \ldots$ & Variation of the useful mirror area & A & 3 & Malfunctions of active mirror control resulting in focusing losses \\
\hline 6.................... & Day-to-day reflectivity changes & A & 2 & Due to dust deposit variations and occasional dew deposit \\
\hline 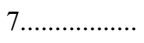 & Photon detection efficiency of the PMT/light catcher system & $\mathrm{A}, \mathrm{C}$ & $10-12$ & See text \\
\hline 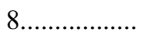 & Unusable camera channels & $\mathrm{B}$ & 3 & Dead PMTs ( $5-10$ channels), problems in calibration (5-10 channels) \\
\hline 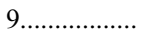 & Trigger inefficiencies & $\mathrm{B}, \mathrm{C}$ & 4 & Due to discriminator dead time, baseline shifts/drifts, level differences trigger branch and FADC branch, etc. \\
\hline $10 \ldots \ldots \ldots \ldots \ldots$ & Signal drift in camera due to temperature drifts & $\mathrm{A}, \mathrm{C}$ & 2 & Combination of PMT QE change (small), amplifier and optical transmitter drifts \\
\hline $11 \ldots \ldots \ldots \ldots . . . . .$. & Camera flat-fielding & A, B & 2 & Calibration problem \\
\hline $12 \ldots \ldots \ldots \ldots \ldots$ & Signal extractor & B & 5 & $\begin{array}{l}\text { Complex effect due to trigger jitter (early pulses from PEs generated on first dynode), etc.; } \\
\text { baseline jitter, shifts in FADCs }\end{array}$ \\
\hline $13 \ldots \ldots \ldots \ldots \ldots$ & Cuts and methods used in the analysis & $\mathrm{B}, \mathrm{C}$ & $5-30$ & Energy dependent; see discussion of differential energy spectrum \\
\hline $14 \ldots \ldots \ldots \ldots \ldots$ & Losses of events during reconstruction & $\mathrm{B}(\mathrm{A})$ & 8 & Simplifications in MC simulation \\
\hline 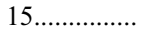 & Estimate of BG under source & $\mathrm{B}(\mathrm{A})$ & 4 & Camera nonuniformity not included in MC; hadronic events not perfectly simulated in $\mathrm{MC}$ \\
\hline $16 \ldots \ldots \ldots \ldots . . . .$. & Small tracking instabilities & $\mathrm{B}$ & 2 & $\begin{array}{l}\text { Source jitters around nominal camera position due to small tracking errors, small camera oscillations } \\
\text { due to gusts, etc., resulting in a wider signal spread than predicted by MC }\end{array}$ \\
\hline $17 \ldots \ldots \ldots \ldots \ldots$ & Nonlinearities in the analog signal chain (PMT-FADC) & $\mathrm{C}(\mathrm{A})$ & $3-10$ & Saturation and nonlinearities of electronic and optoelectronic components \\
\hline
\end{tabular}

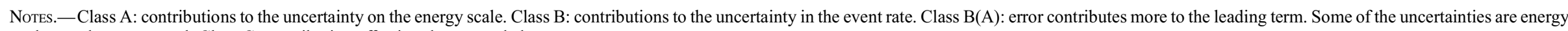
dependent and are averaged. Class $\mathrm{C}$ : contribution affecting the spectral slope. 


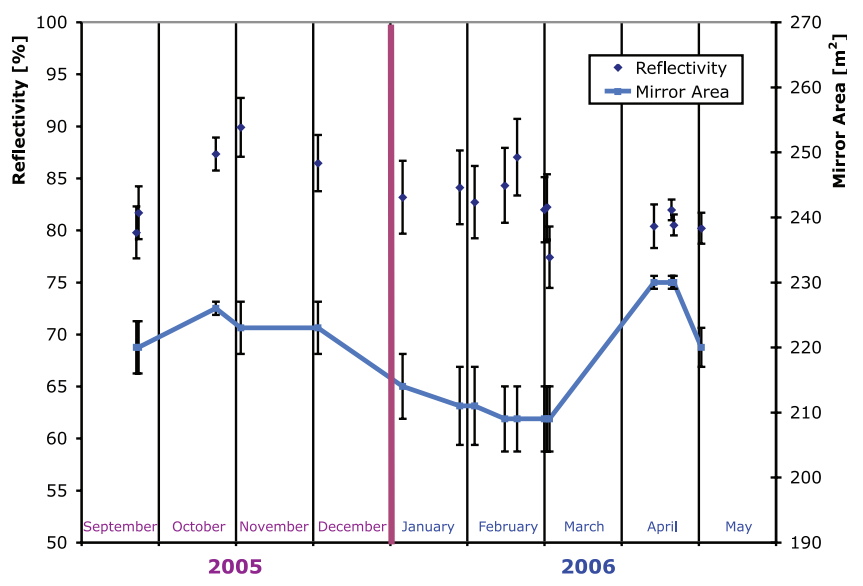

FIG. 7.- Measurement of the reflectivity of the main mirror; the effective mirror area is shown by the blue points (Garczarczyk 2006). The measurements cover the Crab observation period from 2005 October to December.

slope of the spectrum (spectral index) $\Gamma^{\prime}$ was tested for various points of the measured spectrum $\Gamma^{\prime}$ :

$$
\begin{gathered}
\Gamma^{\prime}(E)=\frac{d \ln (F)}{d \ln (E)} \approx \frac{\Delta \ln (F)}{\Delta \ln (E)} \approx \frac{\ln F_{i}-\ln F_{j}}{\ln E_{i}-\ln E_{j}}, \\
E=\exp \left[0.5\left(\ln E_{i}+\ln E_{j}\right)\right],
\end{gathered}
$$

where $F_{i, j}$ is the differential flux measured at $E_{i, j}$. The four derived spectral indices at $\sim 150 \mathrm{GeV}, \sim 300 \mathrm{GeV}, \sim 1 \mathrm{TeV}$, and $\sim 2.5 \mathrm{TeV}$ shown in Figure 12 indicate a clear softening of the spectrum with increasing energy. The spectral index $\Gamma^{\prime}$ was also derived from the aforementioned results of the curved powerlaw fit,

$$
\Gamma^{\prime}=a+2 b \log _{10}(E / 300 \mathrm{GeV})
$$

and is shown by the solid black line, and the $\pm 1 \sigma$ confidence band is shown by the dashed black lines. A systematic uncertainty on the slope can cause an additional vertical shift of the measurement by \pm 0.2 . Within uncertainties, the measured spectral index varies in good agreement with predictions by Aharonian et al. (2004) (blue line), who, in addition to the IC scattering on synchrotron photons, included several other soft photon fields such as millimeter photons, CMB, and far-IR photons from dust and stars.

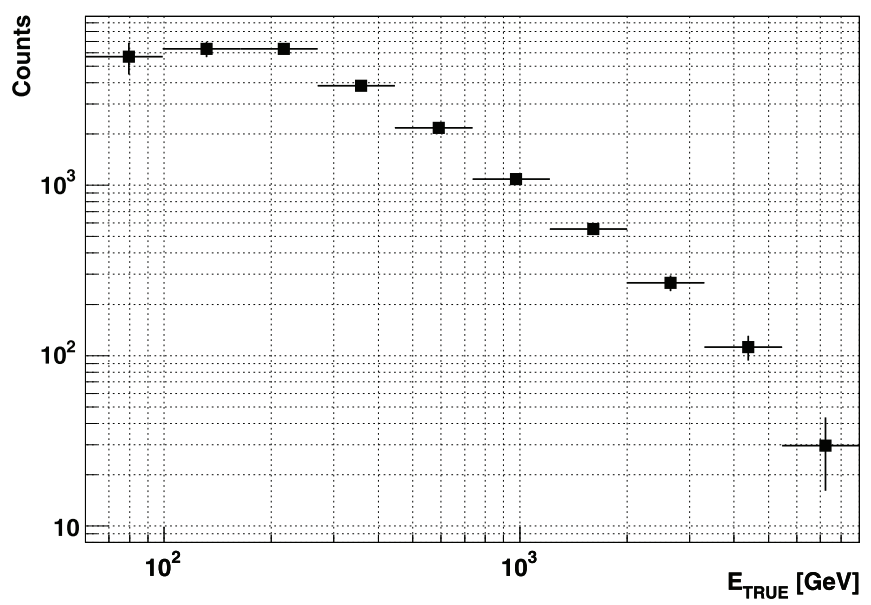

FIG. 8.-Unfolded distribution of excess events from the Crab Nebula. The integral rate of excess events is $0.4 \mathrm{~Hz}$.

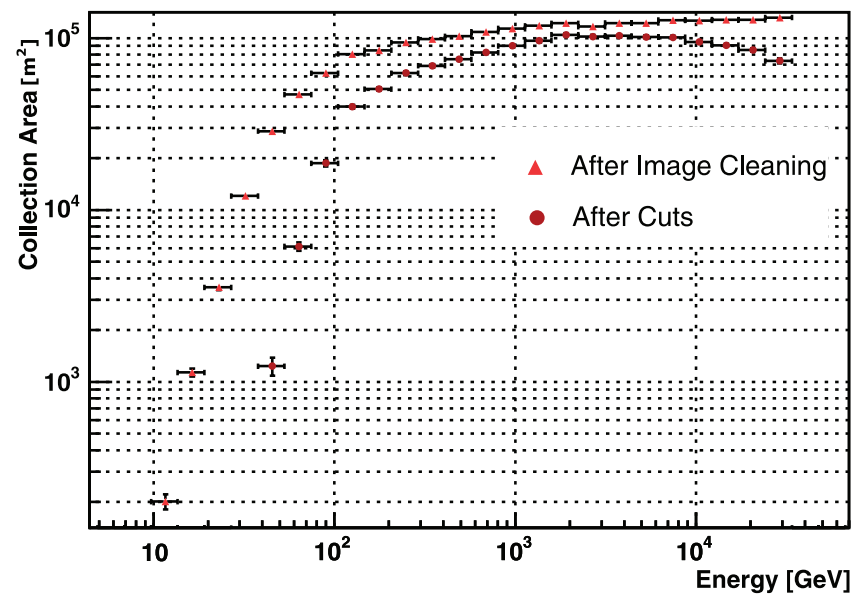

FIG. 9.-Collection area after image cleaning and after cuts for low zenith angle observations $\left(<20^{\circ}\right)$.

The predicted $\mathrm{GeV} \gamma$-ray emission has a peak in the SED representation (see Fig. 11). If one assumes that the energy spectrum around the peak can be described with a curved power law, the position of the peak can be determined from the measurement of the spectral index obtained from the result of the curved powerlaw fit. A necessary condition for the peak in the SED is that the spectral index $\Gamma^{\prime}$ is -2 . With this condition the peak is determined at $77 \pm 47_{\text {stat }}{ }_{-46}^{+107}$ syst $\mathrm{GeV}$ (Fig. 12, triangle).

\subsection{VHE $\gamma$-Ray Light Curve of the Nebula Emission}

In the VHE $\gamma$-ray astronomy community it is assumed that the Crab Nebula is a constant and stable $\gamma$-ray source and can therefore be used as a standard candle. However, with more sensitive measurements it is necessary to check the stability of the $\gamma$-ray source. Below we present a time-resolved measurement of the VHE $\gamma$-ray flux, i.e., the light curve for the Crab Nebula. Depending on the source strength and the analysis threshold, the time intervals can be as short as a few minutes.

We calculated light curves in bins of 10 minutes from events with estimated energies above $200 \mathrm{GeV}$. The light curves of all 14 selected nights are shown in Figure 13. Note that the same loose cuts are used for the $\gamma /$ hadron separation as for the calculation of the energy spectrum, which reduces the sensitivity of the measurement. The probability that the light curve is described by a

TABLE 5

\begin{tabular}{|c|c|}
\hline $\begin{array}{l}\text { Mean Energy } \\
(\mathrm{GeV})\end{array}$ & $\begin{array}{l}\text { Differential Flux } \\
\left(\mathrm{cm}^{-2} \mathrm{~s}^{-1} \mathrm{TeV}^{-1}\right)\end{array}$ \\
\hline .................. & $\left(1.14 \pm 0.27_{\text {stat }} \pm 0.34_{\text {syst }}\right) \times 10^{-8}$ \\
\hline $127 \ldots$. & $\left(3.65 \pm 0.38_{\text {stat }} \pm 0.55_{\text {syst }}\right) \times 10^{-9}$ \\
\hline $210 \ldots \ldots \ldots \ldots \ldots \ldots \ldots$ & $\left(1.41 \pm 0.09_{\text {stat }} \pm 0.28_{\text {syst }}\right) \times 10^{-9}$ \\
\hline $346 \ldots \ldots \ldots \ldots \ldots \ldots \ldots \ldots$ & $\left(4.37 \pm 0.23_{\text {stat }} \pm 0.87_{\text {syst }}\right) \times 10^{-10}$ \\
\hline 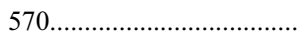 & $\left(1.32 \pm 0.07_{\text {stat }} \pm 0.20_{\text {syst }}\right) \times 10^{-10}$ \\
\hline 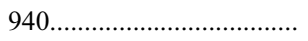 & $\left(3.55 \pm 0.23_{\text {stat }} \pm 0.18_{\text {syst }}\right) \times 10^{-11}$ \\
\hline $1550 \ldots$ & $\left(9.88 \pm 0.74_{\text {stat }} \pm 0.49_{\text {syst }}\right) \times 10^{-12}$ \\
\hline $2554 .$. & $\left(2.69 \pm 0.29_{\text {stat }} \pm 0.27_{\text {syst }}\right) \times 10^{-12}$ \\
\hline . & $\left(6.80 \pm 1.10_{\text {stat }} \pm 1.00_{\text {syst }}\right) \times 10^{-13}$ \\
\hline . & $\left(1.15 \pm 0.53_{\text {stat }} \pm 0.12_{\text {syst }}\right) \times 10^{-13}$ \\
\hline
\end{tabular}

Mean Energy and Differential Flux of the Spectral Points Shown in Figure 10

Note.-The systematic errors are derived from different applied cuts and unfolding procedures. 


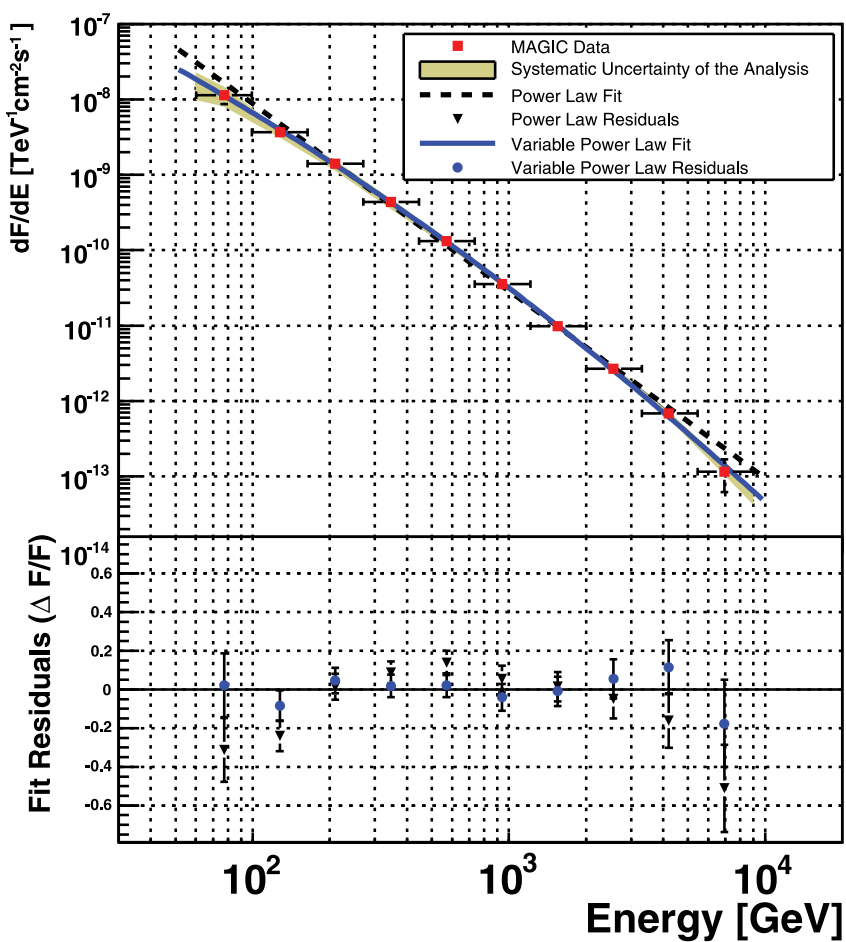

FIG. 10.-Differential energy spectrum of the Crab Nebula. The spectrum was unfolded with the method of Bertero (1989). The results of a fit of the spectrum with a power law and a broken power law are also shown. The bottom panel shows the relative residuals between the fit and the data points. See text for further discussion.

constant flux level is $>10 \%$ in all nights except the first night, where the probability of the fit is $0.8 \%$. The average statistical uncertainty of each flux measurement is $\sim 20 \%$. Figure 14 shows the average flux of each night. The dashed line in the figure denotes the average flux from all nights, and the shaded region shows the statistical error in the flux. The average integral mean flux $F_{>200 \mathrm{GeV}}$ is

$$
F_{>200 \mathrm{GeV}}=\left(1.96 \pm 0.05_{\text {stat }}\right) \times 10^{-10} \mathrm{~cm}^{-2} \mathrm{~s}^{-1} .
$$

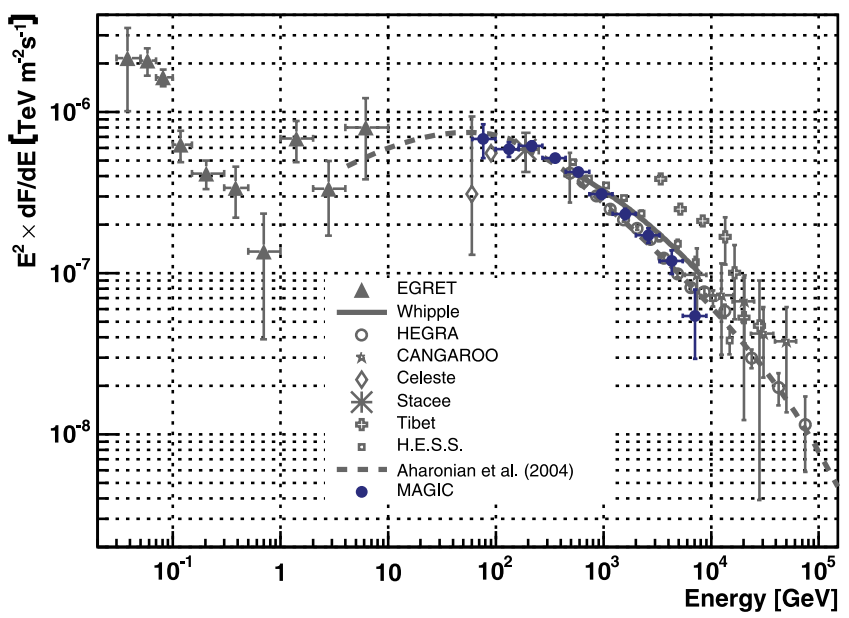

FIG. 11.-SED of the $\gamma$-ray emission of Crab Nebula. The measurements shown below $10 \mathrm{GeV}$ are by EGRET (de Jager et al. 1996). In VHE $\gamma$-rays, measurements are from ground-based experiments. Above $400 \mathrm{GeV}$ our measurement is in agreement with measurements by other IACTs. The dashed line is a model prediction by Aharonian et al. (2004).

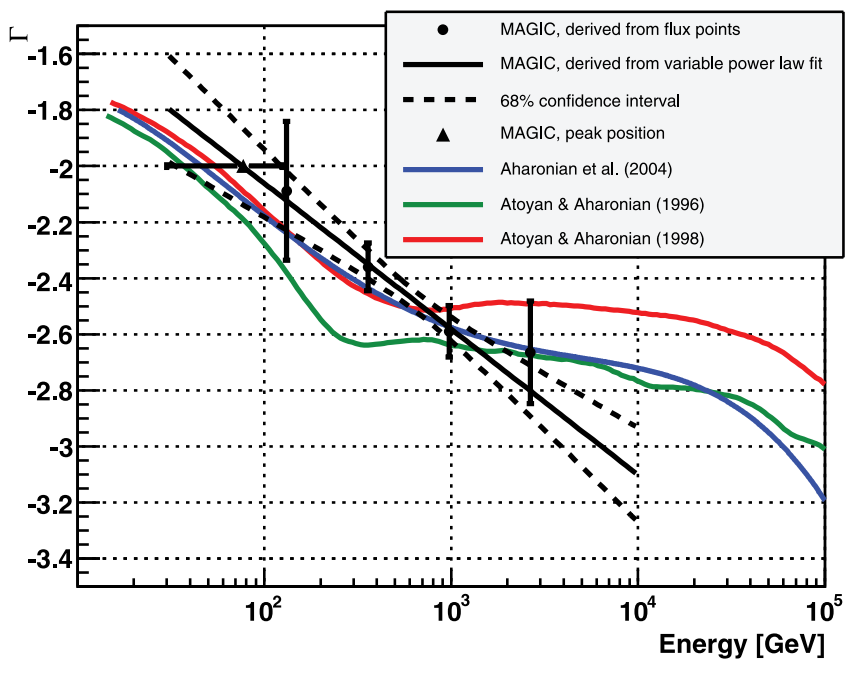

FIG. 12.- Measured spectral index derived from differential flux points ( filled circles) and from the curved power-law fit (black solid line; the dashed line gives the $1 \sigma$ confidence interval). Predictions by Aharonian et al. (2004) (blue line), Atoyan \& Aharonian (1996) (green line), and Aharonian \& Atoyan (1998) (red line) are also shown.

There is a probability of $67 \%$ that the measured daily flux values are compatible with a constant flux. We can, therefore, conclude that the reconstructed flux of the Crab Nebula, within statistical uncertainties, was constant over the entire observation period.

\subsection{Morphology of the $\gamma$-Ray-emitting Region}

The morphology of the $\gamma$-ray emission was studied by generating sky maps in three uncorrelated bins of SIZE. The reconstruction of the origin of a $\gamma$-ray event with a single telescope is possible with the so-called DISP method (Fomin et al. 1994; Lessard et al. 2001). For the studies presented here we used the following parameterization for DISP:

$$
\mathrm{DISP}=a(\mathrm{SIZE})+b(\mathrm{SIZE}) \frac{\mathrm{WIDTH}}{\mathrm{LENGTH}},
$$

where $a$ and $b$ are second-order polynomials found by fitting MC simulated $\gamma$-ray showers (Domingo-Santamaría et al. 2005). Strong tail cuts of 10 and 8 phe were used in the image cleaning for core and boundary pixels, respectively, and a tight HADRONNESS cut $<0.1$ was applied, resulting in improved angular resolution.

The reconstructed event origins were corrected for possible mispointing by using the information from the starguider camera. Two-dimensional (2D) histograms with bin sizes of $0.057^{\circ} \times$ $0.057^{\circ}$ were filled with the corrected event origins (events with energies $<500 \mathrm{GeV}$ ). A 4 times finer binning was chosen for the sky map filled by events with energies above $500 \mathrm{GeV}$. Figure 15 shows the background-subtracted sky maps of excess events from the Crab Nebula for $\gamma$-ray energies $\sim 160, \sim 250$, and $>500 \mathrm{GeV}$.

\subsubsection{Center of Gravity of the $\gamma$-Ray Emission}

The CoG of the $\gamma$-ray emission was derived from the sky maps of the excess events shown in Figure 15 by fitting them with a 2D Gaussian of the form

$$
F(x, y)=F_{\text {res }}+a \exp \left[-\frac{(x-\bar{x})^{2}+(y-\bar{y})^{2}}{2 \sigma^{2}}\right],
$$



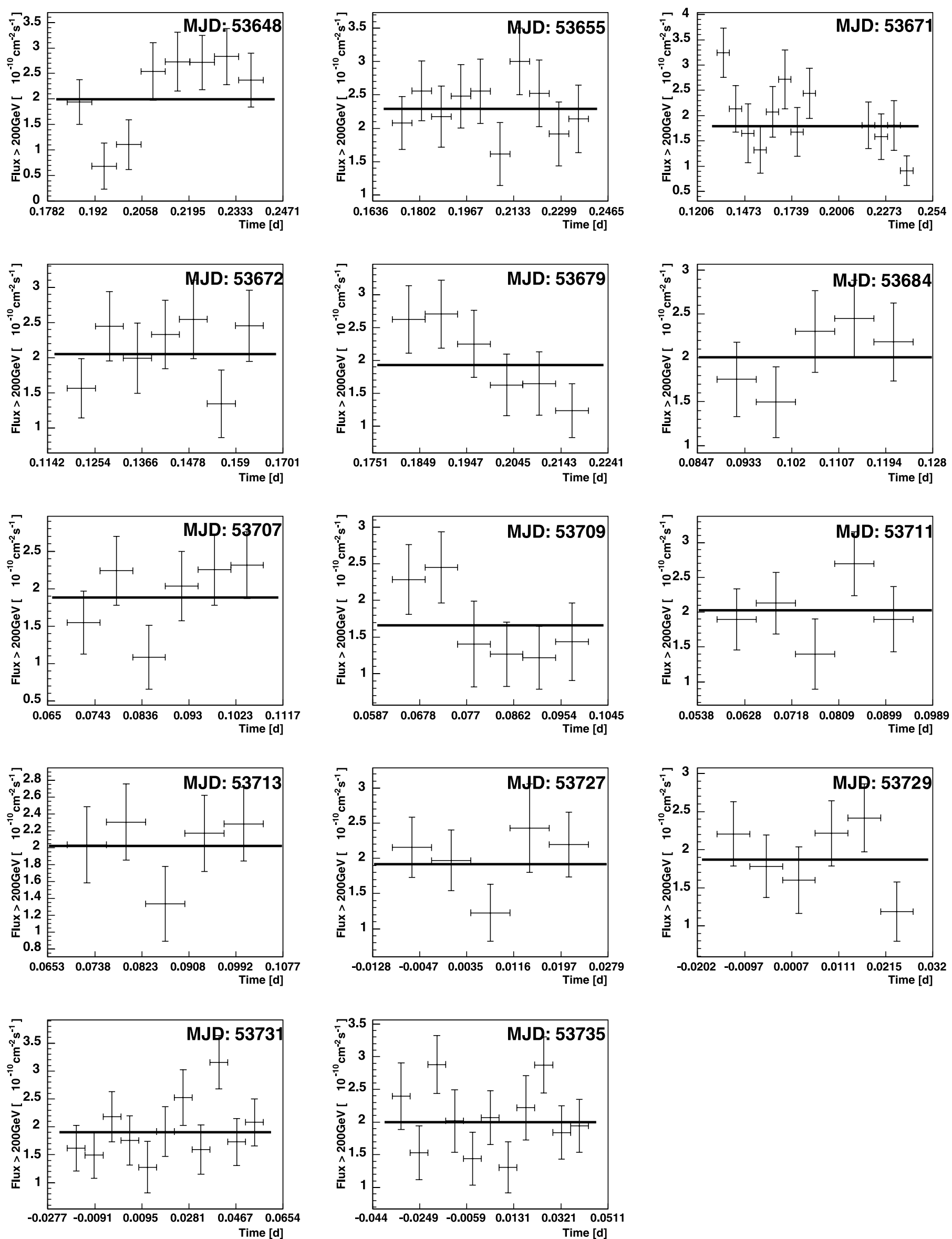

FIg. 13.-Light curves of the integral flux above $200 \mathrm{GeV}$ from the Crab Nebula for each night. 


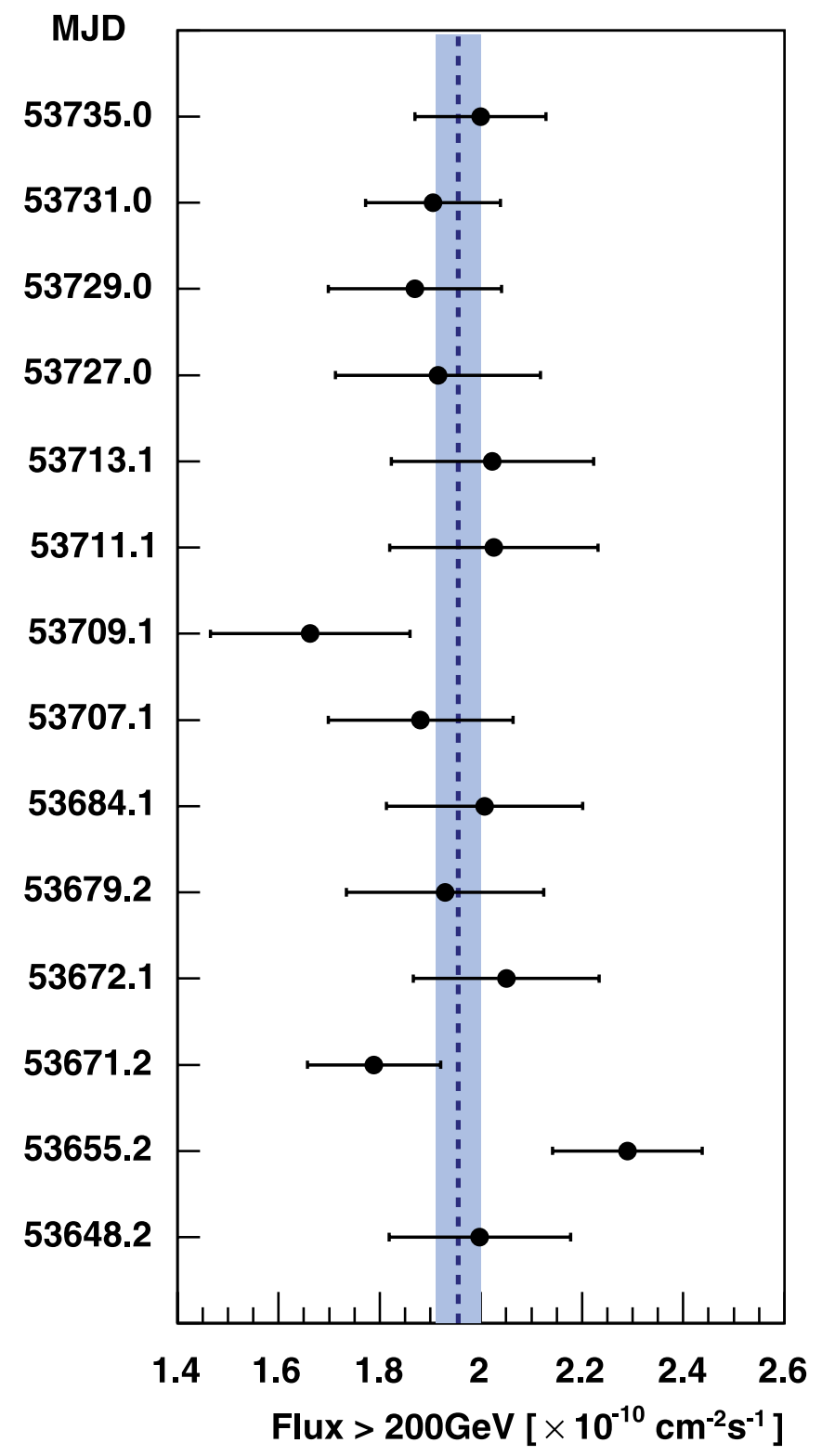

FIG. 14.-Nightly average flux from the Crab Nebula above $200 \mathrm{GeV}$ of each observed night. The dashed blue line gives the average flux of all nights, and the blue shaded region gives the corresponding statistical error.

where $F_{\text {res }}$ is introduced to account for a possible constant offset of the background-subtracted sky map. In this representation $\sigma$ defines the $39 \%$ containment radius of the observed $\gamma$-ray emission. Here we assume that the distribution of excess events is rotationally symmetric, i.e., $\sigma_{x}=\sigma_{y}=\sigma$. It is further assumed that $\sigma$ is the convolution of the response of the detector $\sigma_{\text {PSF }}$ (point-spread function) and the apparent size of the $\gamma$-ray emission region $\sigma_{\mathrm{src}}$, i.e., $\sigma^{2}=\sigma_{\mathrm{PSF}}^{2}+\sigma_{\mathrm{src}}^{2}$. Note that here it is assumed that the $\gamma$-ray emission region follows the shape described by equation (8), which in reality is not necessarily the case.

The CoGs obtained from the fitted $\bar{x}$ and $\bar{y}$ are listed in Table 6 and shown in Figure 16 superimposed on the composite image of optical, IR, and X-ray observations of the Crab Nebula. The three measured CoGs are compatible among each other and coincide with the position of the pulsar. Note that the systematic uncertainty of the position is $\sim 1^{\prime}$.

\subsubsection{Extension of the $\gamma$-Ray Emission Region}

The extension of the $\gamma$-ray emission region was studied by comparing the width of the excess event distribution with that obtained for a simulated $\gamma$-ray point source. The simulated distributions were verified by comparing them to the distributions extracted from an observation of Mrk 421, an extragalactic $\gamma$-ray source that can be considered a point source for our purpose. The Mrk 421 data set is the same as in Albert et al. (2007a). The width of the $\gamma$-ray excess extracted from Mrk 421 and the simulated width for a point source agree within statistical uncertainties.

In the following, the average position of the CoGs obtained from the three sky maps is assumed as the $\gamma$-ray source position. The angular distance squared $\left(\theta^{2}\right)$ between the reconstructed origin and the assumed source position is calculated for every event. The background-subtracted $\theta^{2}$ distributions obtained for the three energy ranges are shown in Figure 17. Data (black) and MC (blue) are compatible within statistical uncertainties in all three $\theta^{2}$ distributions.

An exponential function of the form

$$
F\left(\theta^{2}\right)=a \exp \left(-\frac{\theta^{2}}{2 \sigma^{2}}\right)
$$

describes the expected $\theta^{2}$ distribution, where $a$ is a normalization and $\sigma$ is the same as in equation (8). Values for $\sigma^{2}$ and $\sigma_{\mathrm{PSF}}^{2}$ found by fitting the corresponding $\theta^{2}$ distributions with equation (9) are shown in Table 7. Upper limits on $\sigma_{\text {src }}$ were calculated with a confidence level of $95 \%$ following the procedure outlined in Yao et al. (2006) for one-sided confidence intervals and Gaussian errors. The results are presented in Table 7. For energies above $500 \mathrm{GeV}$ the limit is shown in Figure 16. The limits obtained for $\gamma$-ray energies above $500 \mathrm{GeV}$ and about $250 \mathrm{GeV}$ constrain the $\gamma$-ray emission to a region within the optical synchrotron nebula.

\subsection{Search for Pulsed $\gamma$-Ray Emission}

Among the most challenging tasks of ground-based $\gamma$-ray experiments is the detection of a pulsar. Several experiments have tried but failed. Currently MAGIC is the only ground-based detector with threshold settings below $100 \mathrm{GeV}$ that is appropriate for a search of pulsed $\gamma$-ray emission from the Crab pulsar. For the data a periodicity analysis was performed after $\gamma /$ hadron separation and selection of events with small $\mid$ ALPHA $\mid$ value. The cuts were chosen by MC simulations to optimize the sensitivity of the analysis. After event selection, the event times ${ }^{24}$ were transformed to the barycenter of the solar system with the TEMPO timing package (Taylor et al. $2000^{25}$ ). Then, the corresponding phase $\phi_{j}$ of the Crab pulsar was calculated for each transformed arrival time $t_{j}$ :

$$
\phi_{j}=\nu\left(t_{j}-t_{0}\right)+\frac{1}{2} \dot{\nu}\left(t_{j}-t_{0}\right)^{2}
$$

where $\nu, \dot{\nu}$, and $t_{0}$ are values of contemporary ephemerides of the Crab pulsar provided by the Jodrell Bank Radio Telescope ${ }^{26}$ (see Table 8). We tested for periodicity with the $H$-test (de Jager

\footnotetext{
24 The time of each event was derived from the time signal of a GPScontrolled rubidium clock with a precision of $\sim 200 \mathrm{~ns}$.

${ }_{25}$ Available at http://www.atnf.csiro.au/research/pulsar/tempo/.

26 See http://www.jb.man.ac.uk/pulsar/crab.html.
} 


\section{0-300 phe}

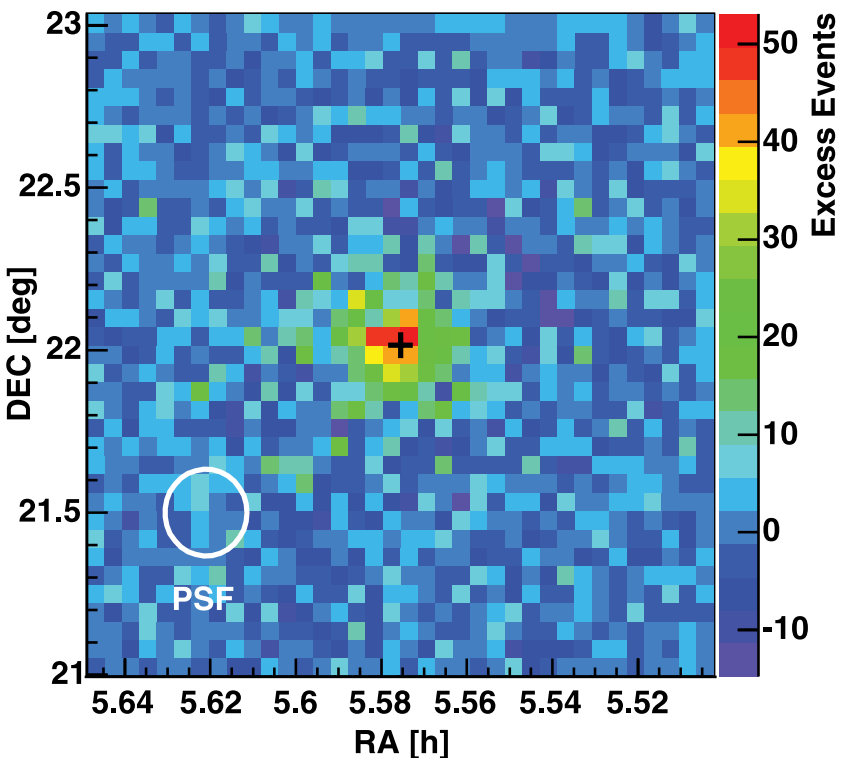

\section{0-700 phe}

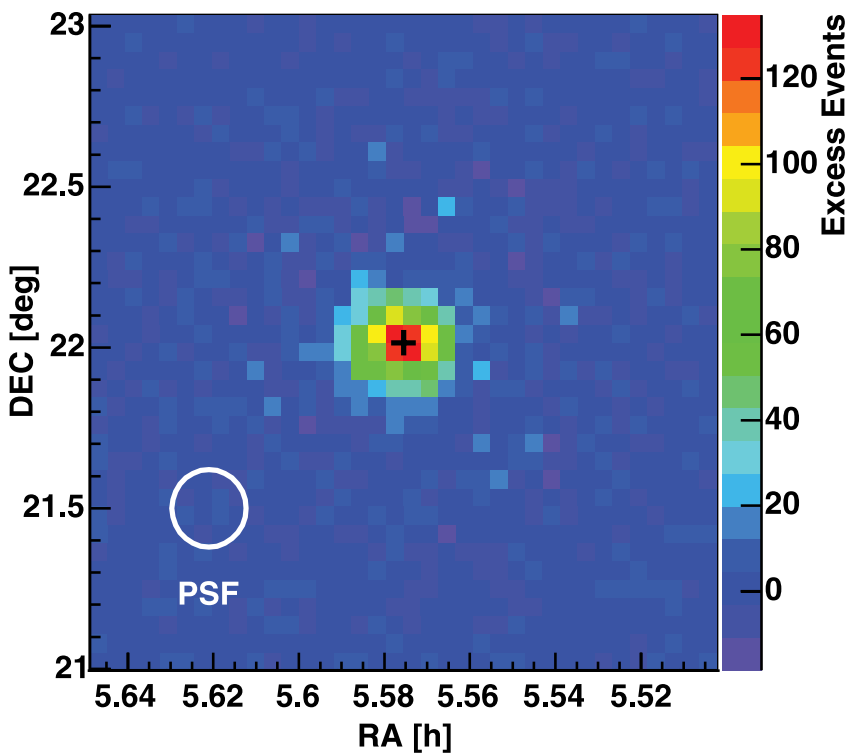

\section{$>700$ phe}

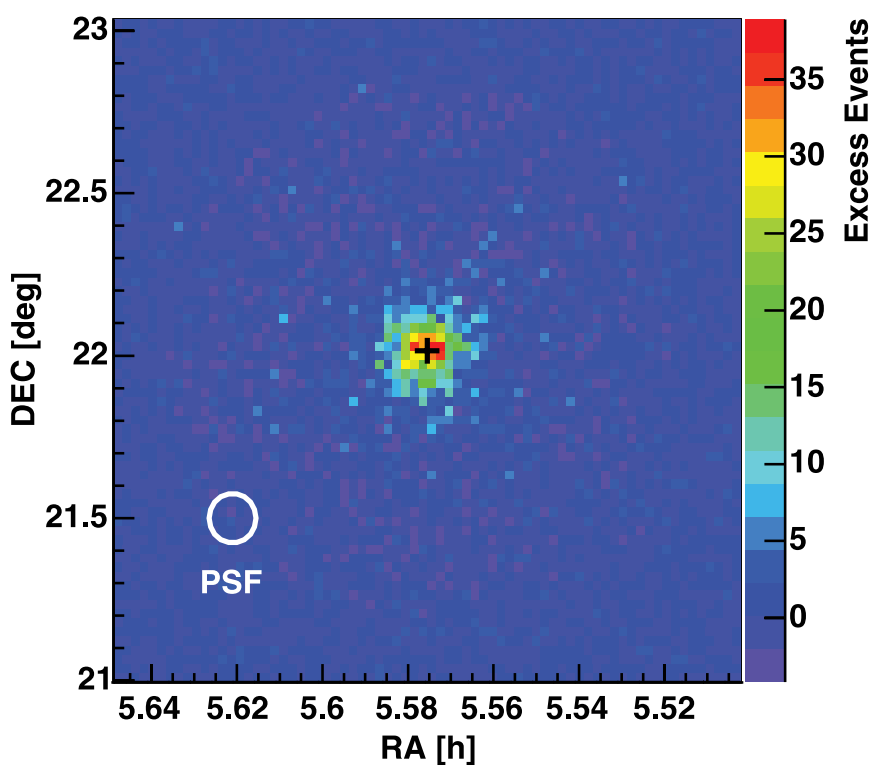

FIG. 15.- Sky maps of excess events from the Crab Nebula for different $\gamma$-ray energies $(\sim 160, \sim 250$, and $>500 \mathrm{GeV})$. The position of the pulsar is marked by the black plus sign, and the angular resolution is indicated by the circle.

TABLE 6

Center of Gravity of the $\gamma$-Ray Emission of the Crab Nebula Obtained For DifFERENT ENERGIES

\begin{tabular}{|c|c|c|c|}
\hline $\begin{array}{l}\text { SIZE } \\
\text { (phe) }\end{array}$ & $\begin{array}{l}\text { Energy } \\
(\mathrm{GeV})\end{array}$ & $\begin{array}{c}\text { Right Ascension } \\
\text { (hr) }\end{array}$ & $\begin{array}{l}\text { Declination } \\
\text { (deg) }\end{array}$ \\
\hline $200-300 .$. & $160_{-50}^{+80}$ & $5.5766 \pm 0.0009$ & $22.019 \pm 0.011$ \\
\hline $300-700 \ldots$ & $250_{-80}^{+130}$ & $5.5758 \pm 0.0003$ & $22.019 \pm 0.004$ \\
\hline 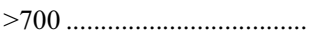 & $>500$ & $5.5759 \pm 0.0003$ & $22.022 \pm 0.003$ \\
\hline Position of the pulsar..... & $\ldots$ & 5.5755 & 22.015 \\
\hline
\end{tabular}

NoTES. - In the first column the applied SIZE cut is stated. The second column shows the corresponding range of $\gamma$-ray energies covered (peak value and FWHM of the distribution of MC $\gamma$-ray events). The last two columns give the fitted position of the CoG and the statistical uncertainty. et al. 1989), the Pearson's $\chi^{2}$-test, and a test from Gregory \& Loredo (1992) that is based on Bayesian statistics.

The analysis chain was tested by optical observations of the Crab pulsar with the MAGIC telescope. Within this $12.5 \mathrm{hr}$ observation, every time the readout of MAGIC was triggered by a cosmic-ray shower, the signal of the pixel in the center of the MAGIC camera was recorded by the MAGIC DAQ for $100 \mathrm{~ns}$. Along with an average trigger rate of $200 \mathrm{~Hz}$, the effective observation time was only about $1 \mathrm{~s}$. Figure 18 shows the reconstructed optical light curve of the Crab pulsar with the familiar main pulse and interpulse. For better readability the light curve is shown twice. The position of the main pulse is shifted with respect to the position of the main pulse in radio by $-252 \pm 64 \mu \mathrm{s}$, which is in agreement with the contemporary measurement of Oosterbroek et al. (2006). 


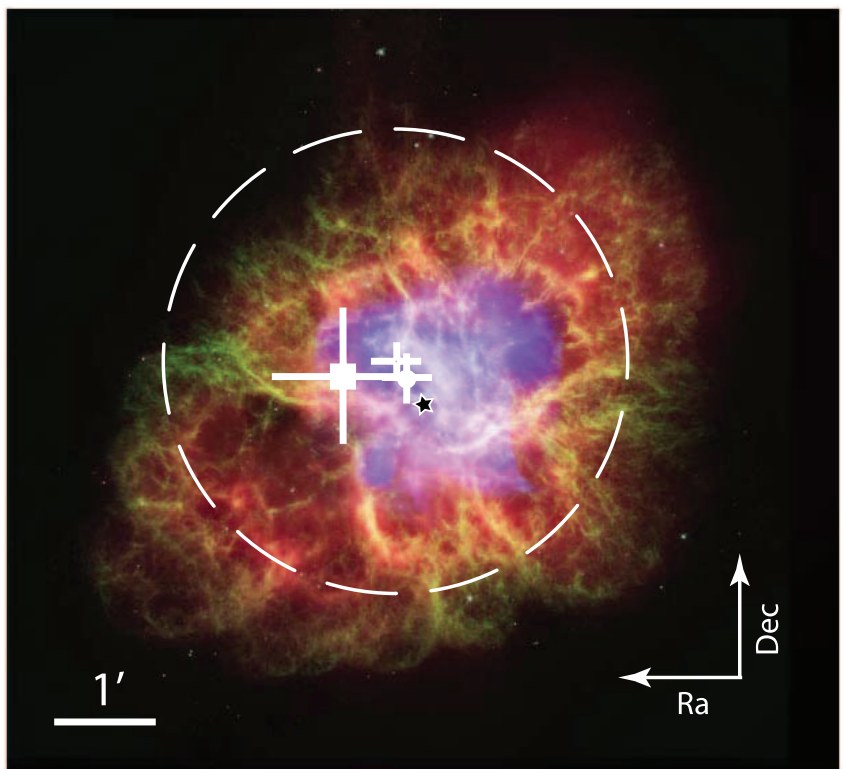

FIG. 16.-Emission of the Crab Nebula in different energy bands. The position of the Crab pulsar is marked with a black star. The Chandra X-ray image is shown in light blue, the Hubble Space Telescope optical images are in green and dark blue, and the Spitzer Space Telescope's infrared image is in red (picture from http://www.chandra.harvard.edu/photo/2006/crab/). Overlaid are the CoG of the $\gamma$-ray emission at different energies ( plus sign: $>500 \mathrm{GeV}$; filled circle: $\sim 250 \mathrm{GeV}$; filled square: $\sim 160 \mathrm{GeV}$ ). The error bars indicate the statistical uncertainty in the position of the CoG. Indicated by the dashed circle is the upper limit $(95 \%$ confidence level) on the $39 \%$ containment radius of the $\gamma$-ray emission region that was derived from the $\theta^{2}$ distribution for $\gamma$-ray energies above $500 \mathrm{GeV}$.

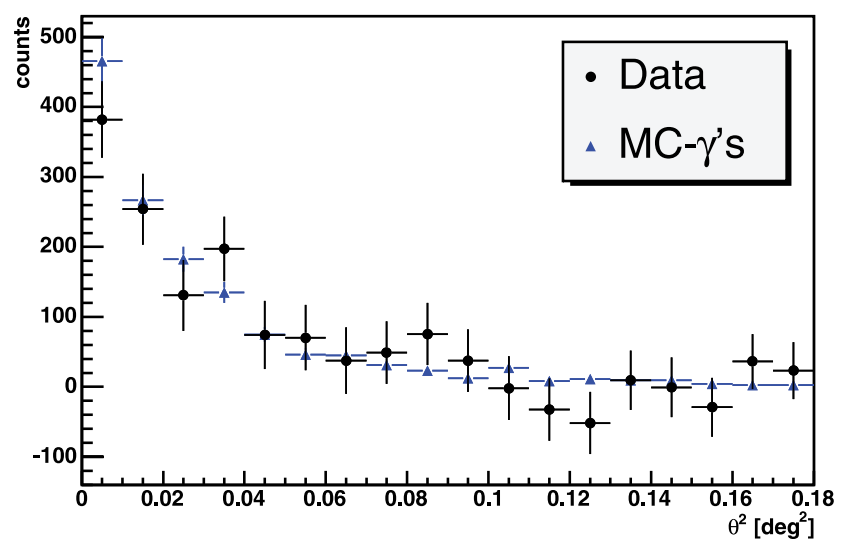

TABLE 7

Results of the Fit of the $\theta^{2}$ Distributions with an Exponential Ansatz and Thereof Derived Upper Limits on the Extension of the Emission Region (39\% Containment Radius)

\begin{tabular}{cccc}
\hline \hline $\begin{array}{c}\text { Energy } \\
(\mathrm{GeV})\end{array}$ & $\begin{array}{c}\text { Data } \sigma^{2} \\
\left(\mathrm{deg}^{2}\right)\end{array}$ & $\begin{array}{c}\mathrm{MC} \sigma_{\mathrm{PSF}}^{2} \\
\left(\mathrm{deg}^{2}\right)\end{array}$ & $\begin{array}{c}95 \% \text { U.L. on Extension } \\
(\operatorname{arcmin})\end{array}$ \\
\hline $160_{-50}^{+80} \ldots \ldots$ & $0.0148 \pm 0.0035$ & $0.0113 \pm 0.0007$ & 5.9 \\
$250_{-80}^{+130} \ldots .$. & $0.0100 \pm 0.0008$ & $0.0100 \pm 0.0003$ & 2.4 \\
$>500 \ldots \ldots .$. & $0.0054 \pm 0.0006$ & $0.0051 \pm 0.0002$ & 2.2 \\
\hline
\end{tabular}

\subsubsection{Search for Pulsed Emission in Differential Bins of Energy}

We searched for pulsed $\gamma$-ray emission in five bins of reconstructed energy between $60 \mathrm{GeV}$ and $9 \mathrm{TeV}$. This search was motivated by a possible pulsed $\gamma$-ray component at $\mathrm{TeV}$ energies (Hirotani 2001, 2007). However, no signature of periodicity was found in any of the tested energy intervals.

For each energy bin an upper limit on the number of excess events was calculated with a confidence level of $95 \%$ in two different ways: first from the result of the $H$-test as described by de Jager (1994), and second from the pulse phase profile. In the calculation of the limit from the result of the $H$-test, it is assumed that the duty cycle (FWHM) of the pulsed $\gamma$-emission is $20 \%$, similar to the duty cycle of the light curve measured by the EGRET detector above $100 \mathrm{MeV}$ (Fierro et al. 1998). No assumption about the position of the emission in the pulse phase profile enters into the calculation. This additional constraint is applied, however, when the upper limit is directly derived from the pulse phase profile. As signal regions we chose the phase intervals where

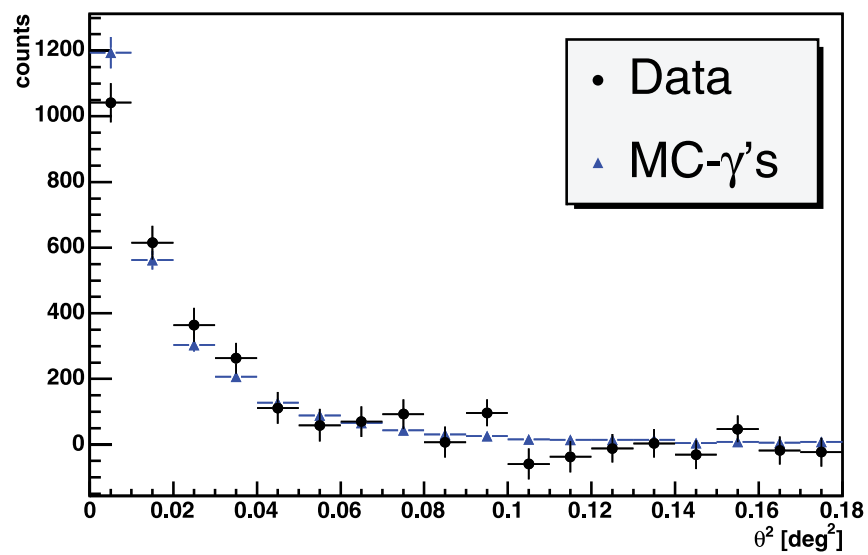

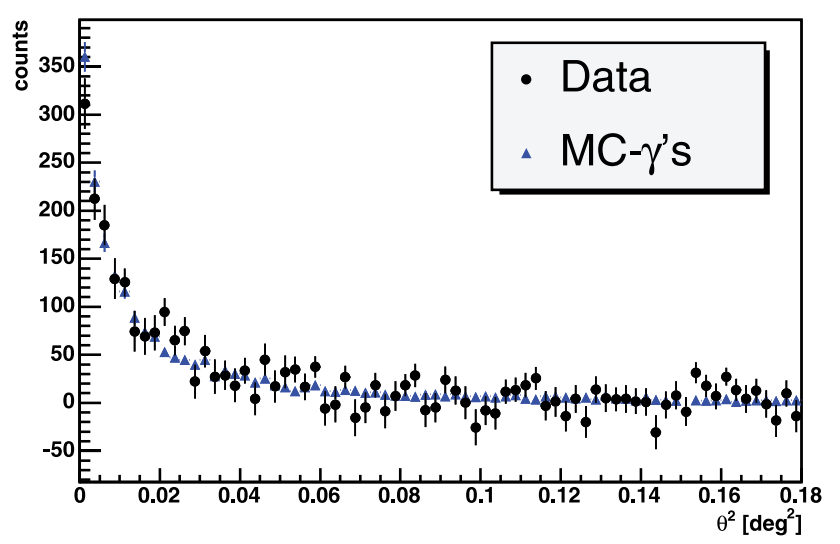

FIG. 17.-Background-subtracted $\theta^{2}$ distributions for different energies: $\sim 160 \mathrm{GeV}$ (top left), $\sim 250 \mathrm{GeV}$ (top right), >500 GeV (bottom). 
TABLE 8

Ephemerides of the Crab Pulsar from the Jodrell Bank Radio Telescope Covering the Same Period of Time as the Analyzed Data

\begin{tabular}{cccc}
\hline \hline JD & $\begin{array}{c}t \\
(\mathrm{~s})\end{array}$ & $\begin{array}{c}\text { Frequency }(\nu) \\
(\mathrm{Hz})\end{array}$ & $\begin{array}{c}\text { Derivative }(\dot{\nu}) \\
\left(10^{-15} \mathrm{~Hz} \mathrm{~s}^{-1}\right)\end{array}$ \\
\hline $2,453,597.5 \ldots \ldots \ldots \ldots$. & 0.029626 & 29.7798524524 & -372992.36 \\
$2,453,628.5 \ldots \ldots \ldots \ldots$. & 0.031767 & 29.7788534525 & -372972.07 \\
$2,453,658.5 \ldots \ldots \ldots \ldots .$. & 0.022656 & 29.7778867428 & -372950.45 \\
$2,453,689.5 \ldots \ldots \ldots \ldots .$. & 0.016803 & 29.7768878849 & -372924.54 \\
$2,453,719.5 \ldots \ldots \ldots \ldots .$. & 0.026788 & 29.7759213143 & -372886.52 \\
$2,453,750.5 \ldots \ldots \ldots \ldots$. & 0.020341 & 29.7749226318 & -372854.62 \\
$2,453,781.5 \ldots \ldots \ldots \ldots .$. & 0.006520 & 29.7739240139 & -372823.70 \\
\hline
\end{tabular}

NotE.-Given in JD is the reference day of the ephemeris, and $t$ is the time of appearance of the first main pulse on the reference day after midnight.

EGRET had observed pulsed emission above $100 \mathrm{MeV}$, i.e., -0.06 to 0.04 and $0.32-0.43$ (shaded region in Fig. 20 below). The background was estimated from the remaining phase intervals. Having defined the signal and background regions in this way, the upper limit on the number of excess events was obtained by the method of Rolke et al. (2005). Because of the additional constraint made about the position of the expected pulsed emission, the limits obtained from the pulse phase profile are on average about a factor of 2 better than the limits obtained from the result of the $H$-test.

The upper limits derived from the pulse phase profiles were converted into flux limits. The collection area was calculated assuming a photon index of -2.6 for the $\gamma$-ray spectrum. The flux limits are shown in Figure 19 together with the upper limit on the cutoff energy, which is derived in the following section.

\subsubsection{Upper Limit on the Cutoff Energy of the Pulsed Emission}

Apart from the search for pulsed emission in bins of reconstructed energy, we performed a periodicity analysis, this time selecting events with SIZE $<300$ phe $(\gamma$-ray energies $\lesssim 180 \mathrm{GeV})$ and applying the same optimized SIZE-dependent HADRONNESS cuts and ALPHA cuts as above. Compared to the previously described analysis, this one is optimized for a search of pulsed emission close to the threshold of the experiment. The analysis threshold, defined as the peak of the energy distribution of simulated $\gamma$-ray showers, is $60 \mathrm{GeV}$.

Figure 20 shows the pulse phase profile obtained for the selected events. For comparison the pulse phase profiles from EGRET observations above $100 \mathrm{MeV}$ and $5 \mathrm{GeV}$ (Thompson et al. 2004) are also shown. The EGRET pulse phase profile above

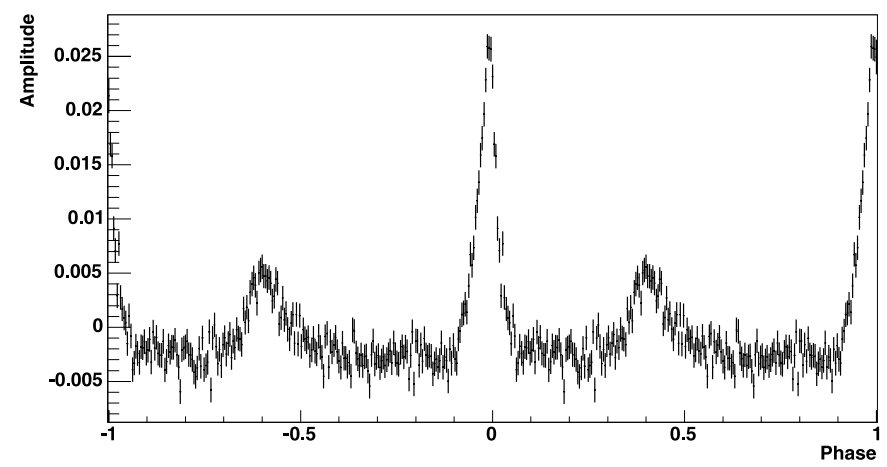

FIG. 18.-Optical light curve of the Crab pulsar measured with MAGIC. The figure includes data from seven different observations between 2005 December and 2006 February. The total observation time was $12.5 \mathrm{hr}$.

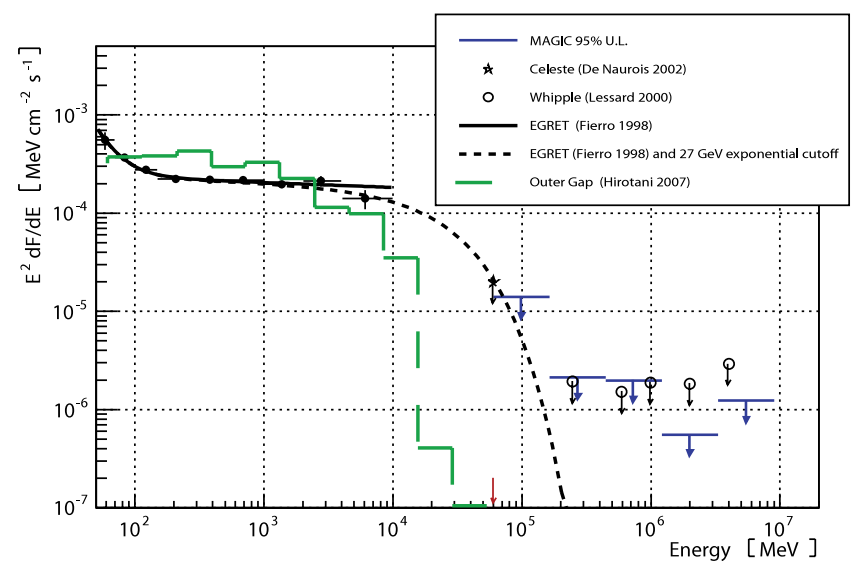

FIG. 19.-Upper limits on the pulsed $\gamma$-ray flux from the Crab pulsar; upper limits in differential bins of energy are given by the blue points. The upper limit on the cutoff energy of the pulsed emission is indicated by the dashed line. The analysis threshold to derive the upper limit on the cutoff energy is indicated by the red arrow.

$10 \mathrm{GeV}$ (Thompson et al. 2005) is not shown because it suffers from too low statistics. Shaded in the pulse phase profiles are the regions of the main pulse and interpulse defined from EGRET observations above $100 \mathrm{MeV}$ (Nolan et al. 1993).

The result of a Pearson's $\chi^{2}$-test is 13.1 with 10 degrees of freedom, corresponding to a significance of $1.2 \sigma$ for periodic emission. The result of the $H$-test is 3.9, which is equivalent to a significance of $1.3 \sigma$. The test by Gregory \& Loredo (1992) results in a probability of $4.1 \times 10^{-4}$ that pulsed emission is present in the data. These tests do not make an assumption about the position of the pulsed emission in the pulse phase profile. However, some evidence of an excess is visible at the position of the interpulse in the same phase range where EGRET detected pulsed emission above $100 \mathrm{MeV}$. If the two phase regions defined by EGRET are used as the signal region and the remaining phase intervals as background region, the significance of the observed excess is $2.9 \sigma$. Note that in this case the significance was not calculated from the binned pulse phase profile shown in Figure 20.

The significance of the observed excess is not sufficient to claim the detection of a pulsed signal; therefore, upper limits on the number of excess events were calculated with a confidence level of $95 \%$ (see Table 9). Note that because of the observed excess, the upper limit from the pulse phase profile is larger than the limit obtained from the $H$-test. Using the different limits on the number of pulsed excess events, we constrain, in the following, the cutoff energy of the pulsar spectrum under the assumption that the break in the energy spectrum can be described with an exponential cutoff. In the procedure we use the parameterization of the measured pulsar spectrum below $10 \mathrm{GeV}$ (Fierro et al. 1998), extended with an exponential cutoff:

$$
\begin{aligned}
F\left(E, E_{\text {cutoff }}\right)= & {\left[7.0 \times 10^{-6}\left(\frac{E}{0.1 \mathrm{GeV}}\right)^{-4.89}\right.} \\
& \left.+2.3 \times 10^{-5}\left(\frac{E}{0.1 \mathrm{GeV}}\right)^{-2.05}\right] \\
& \times \exp \left(-\frac{E}{E_{\text {Cutoff }}}\right) \text { photons }\left(\mathrm{cm}^{2} \mathrm{~s} \mathrm{GeV}\right)^{-1}
\end{aligned}
$$


TABLE 9

Analysis Results for a Cut Selecting Events with SIZE $<300$ phe

\begin{tabular}{|c|c|c|}
\hline Method & $H$-Test & Rolke \\
\hline Test result.. & 3.92 & .. \\
\hline 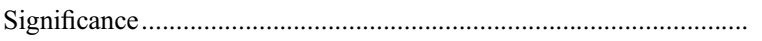 & $1.26 \sigma$ & $\ldots$ \\
\hline $2 \sigma$ U. L. on excess events.. & 1635 & 3198 \\
\hline U. L. on the Cutoff energy $(\mathrm{GeV}) \ldots . .$. & 27 & 34 \\
\hline $2 \sigma$ integral flux limit above $60 \mathrm{GeV}\left(\mathrm{cm}^{-2} \mathrm{~s}^{-1}\right)$. & $2.5 \times 10^{-11}$ & $7.9 \times 10^{-11}$ \\
\hline $2 \sigma$ differential flux limit at $60 \mathrm{GeV}\left(\mathrm{cm}^{-2} \mathrm{~s}^{-1} \mathrm{GeV}^{-1}\right) \ldots \ldots \ldots \ldots \ldots \ldots$ & $4.5 \times 10^{-12}$ & $8.9 \times 10^{-12}$ \\
\hline Peak energy MC $(\mathrm{GeV})$ & 60 & 60 \\
\hline
\end{tabular}

The spectrum with a given $E_{\text {cutoff }}$ is convoluted with the effective collection area after cuts. The collection area is derived from $\mathrm{MC}$ simulations, assuming the same $\gamma$-ray spectrum. The number of expected excess events for the assumed cutoff energy is obtained by multiplying the convoluted spectrum with the observation time. In an iterative algorithm $E_{\text {cutoff }}$ is changed until the number of expected excess events matches the upper limit
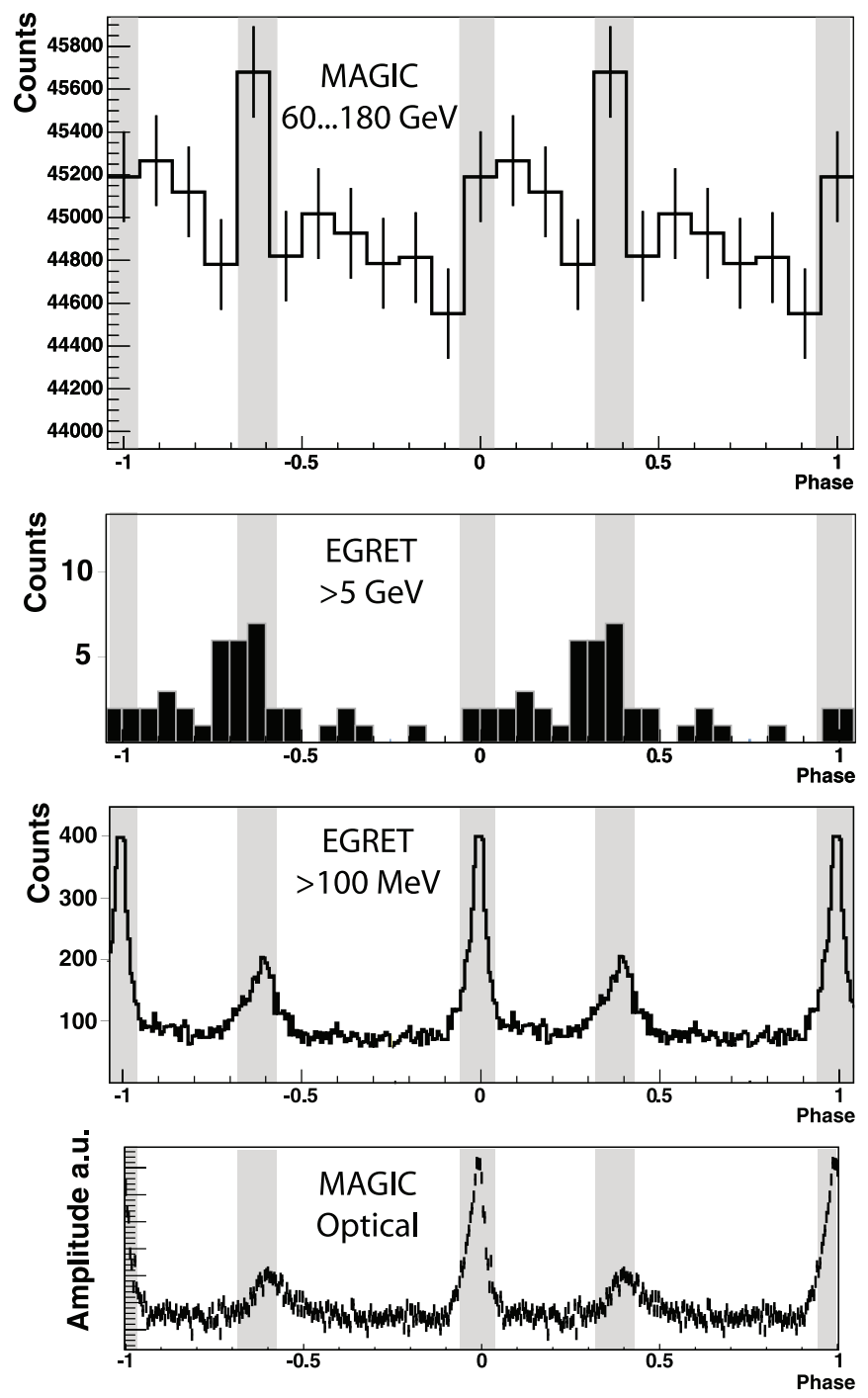

FIG. 20.-Pulse phase profiles of the Crab pulsar. Bottom panel: optical observations by MAGIC ( $\sim 1 \mathrm{~s}$ effective observation time); middle panels: observations by EGRET from Thompson et al. (2004); top panel: pulse phase profile obtained by MAGIC, analysis threshold $60 \mathrm{GeV}$. The shaded regions indicate the EGRET measured positions of the peaks for $\gamma$-ray energies above $100 \mathrm{MeV}$. Note that each pulse phase profile is shown twice for better visibility. on the number of excess events. In this way we derive an upper limit on the cutoff energy of $27 \mathrm{GeV}$ from the result of the $H$-test and $34 \mathrm{GeV}$ from the limit obtained from the pulse phase profile. Differential and integral upper limits on the flux were calculated and are shown in Table 9.

\section{DISCUSSION}

In this paper we report on the most detailed study to date of VHE $\gamma$-ray emission of the Crab Nebula below $500 \mathrm{GeV}$. This study includes the following:

1. A measurement of the differential energy spectrum down to $60 \mathrm{GeV}$.

2. An estimate of the peak in the SED of the VHE $\gamma$-ray emission.

3. The search for an extended source morphology.

4. The calculation of a light curve of the VHE $\gamma$-ray emission from the nebula above $200 \mathrm{GeV}$.

5. A search for pulsed emission from the Crab pulsar in differential bins of energy and in an optimized low-energy analysis.

Most of the aforementioned studies were done in this energy region for the first time; they were possible only because the imaging air shower Cerenkov technique was used. The wave front sampling technique, which, up to now, was the only experimental technique used in this energy domain, did, at best, allow one to arrange an integral flux measurement and to search for pulsed emission. The performance of MAGIC is superior even in the energy domain below $200 \mathrm{GeV}$, where a progressive degradation of the $\gamma /$ hadron separation power is observed. In this context studies for improving the suppression of background events by exploiting the intrinsic time structure of the recorded PMT signals are ongoing. Further improvement is expected with the second MAGIC telescope currently under construction.

The measured energy spectrum of the Crab Nebula in Figure 10 extends over two decades in energy and five decades in flux. The spectral shape deviates from a pure power-law behavior and is, within experimental uncertainties, in agreement with a curved power law. The observation supports the generally accepted picture that the steady emission above a few tens of $\mathrm{GeV}$ and up to the highest measured energies can be described within the framework of the SSC model (Gould 1965; de Jager \& Harding 1992; Aharonian et al. 2004). Also the peak position of the inverse Compton emission of the tested predictions is in agreement with the estimated peak in the SED $\left(77 \pm 47_{\text {stat }}+46\right.$ syst $\left.\mathrm{GeV}\right)$.

At $\mathrm{GeV}$ energies EGRET observed a $\gamma$-ray flux, which was a factor of 5 above the flux predicted by the SSC mechanism (de Jager et al. 1996). Atoyan \& Aharonian (1996) explain this $\gamma$-ray excess by an additional $\gamma$-ray component from bremsstrahlung of electrons that are partially captured in filaments of the nebula. Such an extra component can significantly change the spectral slope at several hundred $\mathrm{GeV}$ compared to a pure IC 
scenario (cf. blue and red lines in Fig. 12) and results in an almost pure power-law behavior of the energy spectrum between $\sim 100 \mathrm{GeV}$ and $10 \mathrm{TeV}$ (constant $\Gamma$ ). At several hundred $\mathrm{GeV}$, where the measurement is most sensitive, the measured slope (black line and data point) is considerably harder than predicted by Atoyan \& Aharonian (1996). It is, therefore, unlikely that the $\gamma$-ray excess at $\mathrm{GeV}$ energies can be explained by bremsstrahlung as proposed. Later predictions by Aharonian \& Atoyan (1998) that also include the mentioned bremsstrahlung mechanism are in agreement with the presented measurement (red line). However, all above-mentioned predictions agree with the measurement if the measured slope is shifted by 0.2 to more negative values, which is within the range of the systematic uncertainty of the measurement. In the prediction by Aharonian \& Atoyan (1998) also a $\gamma$-ray component from $\pi^{0}$-decay is included, which results in a considerable harder spectrum above a few $\mathrm{TeV}$ compared to the pure IC scenario (cf. Fig. 12). However, given the limited statistics above $1 \mathrm{TeV}$ of our measurement, one cannot exclude any such prediction from the measurement.

Studies about the morphology of the $\gamma$-ray-emitting region of the Crab Nebula have been performed by Aharonian et al. (2000, 2004) for $\gamma$-ray energies above $1 \mathrm{TeV}$. In both cases it was found that within the resolution of the experiment the emission region is pointlike. They placed an upper limit on the source size of $\sim 2^{\prime}$ at energies between 1 and $10 \mathrm{TeV}$. In the VHE domain, the morphology of the emission region has not yet been studied at energies below $1 \mathrm{TeV}$. With the resolution of MAGIC it was possible to constrain the origin of the $\gamma$-ray emission to be within the optical synchrotron nebula (see Fig. 16). The upper limit on the size of the emission region is $\sim 2^{\prime}$, which is about 4 times larger than the predicted size of the inverse Compton surface brightness for $\gamma$-ray energies below $500 \mathrm{GeV}$ (de Jager \& Harding 1992).

$\mathrm{X}$-ray observations indicate variabilities in the acceleration and cooling times of electrons on timescale of months (e.g., Hester et al. 2002). However, variations in $\gamma$-rays could not be detected so far. The sensitivity of MAGIC allowed us to study the variability of the $\gamma$-ray emission above $150 \mathrm{GeV}$ on timescales as short as a few minutes up to months. We measured a flux that is within statistics compatible with steady emission. During the observation the stability of the integral flux was better than $10 \%$ on all tested timescales.

In a search for pulsed VHE $\gamma$-ray emission with MC optimized cuts in HADRONNESS and ALPHA an excess was found in the pulse phase profile at the same position where EGRET detected pulsed emission above $100 \mathrm{MeV}$. The significance of the excess is
$2.9 \sigma$ if the phase regions where EGRET detected pulsed emission were chosen as signal regions and the remaining phase intervals are considered as background regions. The similarity of the distribution of excess events in the EGRET $>5 \mathrm{GeV}$ and MAGIC data and the monotonic increase of the number of excess events with increasing upper SIZE cut are strong indications that the observed excess is not a random fluctuation.

With the result of the $H$-test an upper limit on the cutoff energy of $27 \mathrm{GeV}$ was derived, assuming that the power-law spectrum of the pulsar at $\mathrm{GeV}$ energies is attenuated by an exponential cutoff. However, if the cutoff of the spectrum has a superexponential shape, a cutoff energy almost as high as the analysis threshold $(\sim 60 \mathrm{GeV})$ cannot be excluded.

With the derived upper limit we constrain not only the $\gamma$-ray emission from within the light cylinder but also the predicted pulsed $\gamma$-ray emission in the unshocked wind region (Bogovalov $\&$ Aharonian 2000). The predicted $\gamma$-ray flux strongly depends (1) on the distance from the light cylinder where the kinetic energydominated wind forms and (2) on the wind's Lorentz factor. By comparing our observational limits on the pulsed emission with the predicted spectra by Bogovalov \& Aharonian (2000), we can exclude the formation of a particle-dominated wind within a few light cylinder radii. Following the argumentation of the same authors, the particle-dominated pulsar wind must therefore be formed farther out, most likely at distances of more than 30 light cylinder radii.

Also, no pulsed emission was detected for energies above $100 \mathrm{GeV}$, which could have its origin in IC upscattering of IR photons within the light cylinder. Despite earlier claims of a strong component (Hirotani \& Shibata 2001), latest models (Hirotani 2007) seem to disfavor a pulsed $\mathrm{TeV}$ component from the Crab pulsar due to dominant $\gamma-\gamma$ absorption processes. In the future, detailed spectroscopic studies of the pulsed emission by, e.g., GLAST and ground-based experiments with lower thresholds and higher sensitivities like MAGIC II (under construction) and CTA (projected) will hopefully resolve the long-standing question of the origin of the pulsed emission.

We are grateful for discussions with Kouichi Hirotani. We also would like to thank the IAC for the excellent working conditions at the ORM in La Palma. The support of the German BMBF and MPG, the Italian INFN, the Spanish CICYT, ETH research grant TH 34/04 3, and the Polish MNiI grant 1P03D01028 is gratefully acknowledged.
Acharya, B. S., Bhat, P. N., Gandhi, V. N., Ramana Murthy, P. V., Sathyanarayana,

G. P., \& Vishwanath, P. R. 1992, A\&A, 258, 412

Aharonian, F. et al. 1999, A\&A, 346, 913

.2004, ApJ, 614, 897

2006, A\&A, 457, 899

Aharonian, F. A., \& Atoyan, A. M. 1998, in Neutron Stars and Pulsars: Thirty

Years after the Discovery, ed. N. Shibazaki (Tokyo: Universal Academy), 439 Aharonian, F. A., et al. 2000, A\&A, 361, 1073

Akerlof, C., Dimarco, J., Levy, H., MacCallum, C., Meyer, D., Radusewicz, P., Tschirhart, R., \& Yama, Z. 1990, in Proc. 21st Int. Cosmic Ray Conf. (Adelaide), 135

Albert, J., et al. 2006, preprint (astro-ph/0612385) . 2007a, ApJ, 663, 125

2007b, Nucl. Instrum. Methods A, 583, 494

2008, Nucl. Instrum. Methods A, in press (arXiv: 0709.3719)

Amato, E., Guetta, D., \& Blasi, P. 2003, A\&A, 402, 827

Amenomori, M., et al. 1999, ApJ, 525, L93

Anykeyev, V. B., Spiridonov, A. A., \& Zhigunov, V. P. 1991, Nucl. Instrum.

Methods Phys. Res. A, 303, 350

Arons, J. 1983, ApJ, 266, 215

\section{REFERENCES}

Arqueros, F., et al. 2002, Astropart. Phys., 17, 293

Atoyan, A. M., \& Aharonian, F. A. 1996, MNRAS, 278, 525

Baillon, P., et al. 1991, in Proc. 22nd Int. Cosmic Ray Conf. (Dublin), 220

Bednarek, W., \& Bartosik, M. 2003, A\&A, 405, 689

Bednarek, W., \& Protheroe, R. J. 1997, Phys. Rev. Lett., 79, 2616

Bertero, M. 1989, Electronics and Electron Phys., 75, 1989

Bhat, P. N., Ramanamurthy, P. V., Sreekantan, B. V., \& Vishwanath, P. R. 1986, Nature, 319, 127

Bock, R. K., et al. 2004, Nucl. Instrum. Methods Phys. Res. A, 516, 511 Bogovalov, S. V., \& Aharonian, F. A. 2000, MNRAS, 313, 504

Breiman, L. 2001, Machine Learning, 45, 5

Bretz, T., \& Wagner, R. M. 2003, in Proc. 5th Int. Cosmic Ray Conf., 2947

Cheng, K. S., Ho, C., \& Ruderman, M. 1986a, ApJ, 300, 500 1986b, ApJ, 300, 522

Chiang, J., \& Romani, R. W. 1992, ApJ, 400, 629

Cocke, W. J., Disney, M. J., \& Taylor, D. J. 1969, Nature, 221, 525

Collins, G. W., II, Claspy, W. P., \& Martin, J. C. 1999, PASP, 111, 871

Cortina, J., et al. 2005, in Proc. 29th Int. Cosmic Ray Conf. (Pune), 359

Daugherty, J. K., \& Harding, A. K. 1982, ApJ, 252, 337

de Jager, O. C. 1994, ApJ, 436, 239 
de Jager, O. C., \& Harding, A. K. 1992, ApJ, 396, 161

de Jager, O. C., Harding, A. K., Michelson, P. F., Nel, H. I., Nolan, P. L., Sreekumar, P., \& Thompson, D. J. 1996, ApJ, 457, 253

de Jager, O. C., Raubenheimer, B. C., \& Swanepoel, J. W. H. 1989, A\&A, 221, 180 de Naurois, M., et al. 2002, ApJ, 566, 343

Domingo-Santamaría, E., et al. 2005, in Proc. 29th Int. Cosmic Ray Conf. (Pune), 363

Downthwaite, J. C., Harrison, A. B., Kirkman, I. W., Macrae, H. J., McComb, T. J. L., Orford, K. J., Turver, K. E., \& Walmsley, M. 1984, ApJ, 286, L35 Fierro, J. M., Michelson, P. F., Nolan, P. L., \& Thompson, D. J. 1998, ApJ, 494, 734

Fomin, V. P., Stepanian, A. A., Lamb, R. C., Lewis, D. A., Punch, M., \& Weekes, T. C. 1994, Astropart. Phys., 2, 137

Garczarczyk, M. 2006, Ph.D. thesis, Univ. Rostock

Gaug, M., Bartko, H., Cortina, J., \& Rico, J. 2005, in Proc. 29th Int. Cosmic Ray Conf. (Pune), 375

Gibson, A. I., Harrison, A. B., Kirkman, I. W., Lotts, A. P., Macrae, J. H., Orford, K. J., Turver, K. E., \& Walmsley, M. 1982, Nature, 296, 833

Goret, P., Palfrey, T., Tabary, A., Vacanti, G., \& Bazer-Bachi, R. 1993, A\&A, 270,401

Gould, R. J. 1965, Phys. Rev. Lett., 15, 577

Gregory, P. C., \& Loredo, T. J. 1992, ApJ, 398, 146

Harding, A. K., Tademaru, E., \& Esposito, L. W. 1978, ApJ, 225, 226

Hester, J. J., et al. 2002, ApJ, 577, L49

Hillas, A. M. 1985, in Proc. 19th Int. Cosmic Ray Conf. (La Jolla), 445

Hillas, A. M., et al. 1998, ApJ, 503, 744

Hirotani, K. 2001, ApJ, 549, 495 2007, ApJ, 662, 1173

Hirotani, K., \& Shibata, S. 2001, ApJ, 558, 216

Kennel, C. F., \& Coroniti, F. V. 1984a, ApJ, 283, 694 1984b, ApJ, 283, 710

Konopelko, A., et al. 1996, Astropart. Phys., 4, 199

Lessard, R. W., Buckley, J. H., Connaughton, V., \& Le Bohec, S. 2001, Astropart. Phys., 15, 1

Lessard, R. W., et al. 2000, ApJ, 531, 942
Li, T.-P., \& Ma, Y.-Q. 1983, ApJ, 272, 317

Lorenz, E. 2004, NewA Rev., 48, 339

Lucarelli, F., et al. 2005, in Proc. 29th Int. Cosmic Ray Conf. (Pune), 367

Majumdar, P., et al. 2002, in The Universe Viewed in Gamma-Rays, ed. R. Enomoto, M. Mori, \& S. Yanagita (Tokyo: Universal Academy)

Mirzoyan, R., \& Lorenz, E. 1997, in Proc. 25th Int. Cosmic Ray Conf. (Durban), 265

Muslimov, A. G., \& Harding, A. K. 2003, ApJ, 588, 430

Musquere, A. 1999, in Proc. 26th Int. Cosmic Ray Conf. (Salt Lake City), 460

Nolan, P. L., et al. 1993, ApJ, 409, 697

Oosterbroek, T., de Bruijne, J. H. J., Martin, D., Verhoeve, P., Perryman, M. A. C., Erd, C., \& Schulz, R. 2006, A\&A, 456, 283

Oser, S., et al. 2001, ApJ, 547, 949

Paneque, D., Gebauer, H. J., Lorenz, E., Martinez, M., Mase, K., Mirzoyan, R., Ostankov, A., \& Schweizer, T. 2003, Nucl. Instrum. Methods Phys. Res. A, 504, 109

Rees, M. J., \& Gunn, J. E. 1974, MNRAS, 167, 1

Rolke, W. A., López, A. M., \& Conrad, J. 2005, Nucl. Instrum. Methods Phys. Res. A, 551, 493

Schmelling, M. 1994, Nucl. Instrum. Methods Phys. Res. A, 340, 400

Smith, D. A., et al. 2006, A\&A, 459, 453

Staelin, D. H., \& Reifenstein, E. C. 1968, Science, 162, 1481

Tanimori, T., et al. 1998, ApJ, 492, L33

Taylor, J. H., Manchester, R. N., Nice, D. J., Weisberg, J. M., Irwin, A., \& Wex, N. 2000, Tempo Pulsar Timing Package

Thompson, D. J., et al. 1999, ApJ, 516, 297

. 2004, in Cosmic Gamma-Ray Sources, ed. K. S. Cheng \& G. E. Romero (Dordrecht: Kluwer), 149 2005, ApJS, 157, 324

Tikhonov, A. N., \& Arsenin, V. J. 1979, Methods of Solution of Ill-posed Problems (Washington: Winston \& Sons)

Vacanti, G., et al. 1991, ApJ, 377, 467

Weekes, T. C., et al. 1989, ApJ, 342, 379

Yao, W.-M., et al. 2006, J. Phys. G, 33, 1 